Copyright C1997, American Institute of Aeronautics and Astronautics, Inc.

AIAA Meeting Papers on Disc, January 1997

A9715677, N000114-92--1750, AIAA Paper 97-0648

\title{
Free-surface turbulent flows
}

\author{
L. P. Bernal \\ Michigan Univ., Ann Arbor \\ J. Scherer \\ Michigan Univ., Ann Arbor
}

\begin{abstract}
AIAA, Aerospace Sciences Meeting \& Exhibit, 35th, Reno, NV, Jan. 6-9, 1997
We review experimental and computational studies of free-surface turbulent jets and report on a recently completed experimental study of the interaction of a coflowing jet with the free surface. The mean flow scaling and the turbulent structure were measured. The vertical structure at the surface is determined using particle image velocimetry. The presence and evolution of the surface current, as well as enstrophy production at the surface, are documented. The results show a complex highly 3D interaction. Enstrophy production at the surface is localized at the location where vortex reconnection processes occur. (Author)
\end{abstract}




\title{
FREE-SURFACE TURBULENT FLOWS
}

\author{
L.P. Bernal* ${ }^{*}$ and J. Scherer ${ }^{\dagger}$ \\ Department of Acrospace Engineering \\ University of Michigan \\ Ann Arbor, ML. 48109-2118
}

\begin{abstract}
We review experimental and computational studies of freesurface turbulent jets and report on a recently completed experimental study of the interaction of a collowing jet with the free surface. The mean flow scaling and the turbulent structure were measured. The vortical structure at the surface was determined using particle image velocimetry (PIV). The presence and evolution of the surface current is documented as well as enstrophy production at the surface. The results show a complex highly three dimensional interaction. Enstrophy production at the surface is localized at the location where vortex rcconnection processes occur.
\end{abstract}

\section{INTRODUCTION}

Free surface turbulence appears in many flows of practical interest and manifests itself most commonly in flows with waterair interfaces. Such a flow occurs in the wake of a ship where a large quantity of turbulent energy is introduced in the presence of a free surface. Synthetic Aperture Radar (SAR) images of the sca surface show distinct features caused by the interaction of the turbulent subsurface flow with the water surface.' Many aspects of this surface signature are poorly understood and can not be predicted or modeled adequately.

One of the simpler configurations where these phenomena can be studied is that of a turbulent water jet issuing parallel and close to a free surface. Figure 1 shows a schematic of such a configuration. In this case the jet is axisymmetric and issues into a coflowing stream. The jet grows with downstream distance and interacts with the surface; the flow induces deformations on the surface and the surface influences the development of the jet. This flow captures many of the turbulent flow processes in the near-field of a ship wake where high-momentum fluid from the propulsor mixes with the viscous wake of the ship hull near the water surface.

Madnia and Bernal' report measurements of the mean flow and surface signature of a free-surface jet. They found a reduction of the decay rate of the maximum velocity due to the presence of the surface. At sufficiently high Froude number

\footnotetext{
* Associate Professor, Scnior Member AIAA.

$\dagger$ Present address: Mercury Marine, 105 Marine Drive, Hart lord, WI 53027, Member AIAA.

Copyright $\bigcirc 1997$ American Institute of Aeronautics and Astronautics, Inc. All rights reserved
}

( $F_{r}=U_{e} / \sqrt{g h}$ ), the interaction of large scale structures in the jet with the surface produces surface waves that propagate in the lateral direction. The amplitude of the surface deformation was characterized by surlace curvature measurements. They also noted the onset of swirls on the free surface associated with vorticity normal to the free surface.

Anthony and Willmarth' report three-component LDV measurements of the mean velocity and turbulent Reynolds stresses in a free-surface jet. They note anisotropy of the normal components of the Reynolds stress near the surface. This effect, which was first observed by Swean et al. ${ }^{4}$ is consistent with a dominance of surface normal vorticity at the free surface. Anthony and Willmarth also identify the presence of a thin outward flow region near the surface that they called "the surface current". The surface current is attributed to surface parallel vorticity interacting with its image and propagating away from the centerline.

Walker, Chen, and Willmarth' have extended the work of Anthony and Willmarth. They also used a three component LDV and measure turbulence statistics at two downstream locations. By using large ensemble sizes they were able to accurately calculate the mean velocity vector and Reynolds stress tensor for selected measurement locations. Cases with two Froude numbers and two Reynolds numbers were considered where these parameters varied by an order of magnitude. Their work confirms the presence of a surface current and attributes its formation to streanwise. surface-tangent vorticity interacting with its image above the surface and propagating outward. This ontward propagation of vorticity increases the tangential velocity fluctuations near the surface at the expense of the surface normal fluctuations causing anisotropy of the Reynolds stress tensor.

At high Froude number, surface disturbances increase and radiate energy away from the flow in the form of waves. This has the effect of reducing the vortex interaction process and damping the surface current. Walker et al.'s show a reduction in turbulent kinetic energy of up to $20 \%$ for the high Froude number case. The effect of increased Reynolds number was shown to slow the growth and decay of the jet with downstream distance.

Mangiavacchi ${ }^{6}$ performed direct numerical simulations of a temporally growing round turbulent free-surface jet. Statistics and turbulence structure were found to be in good agreement with the previous experimental results. The numerical results show the anisotropy of the turbulent fluctuations near the free surface and the generation of the surface current. Both surface normal and surface tangent vortex structures were identified. For 
the surface normal vorticity, a reverse energy cascade was sometimes present near the free surface as evidenced by merging of vortices and their growth to larger scales. This has the effect of ereating a nearly two-dimensional turbulence field near the free surface and is consistent with the experimentally observed reduction in surface normal velocity tluctuations. This two dimensional turbulence behavior at the free surface was observed by Gharib et al.' in an investigation of grid generated turbulence at a free surface. However, recent direct numerical simulations by Walker et al." of shear-free turbulence near a free surface does not show an increase of two dimensionality as the free surface is approached. Of course the evolving jet flow studied in the present experiments is highly three dimensional and enhanced two dimensionality associated with surface normal vorticity is not expected to play an important role in the dynamics of the flow.

Walker and Chen" evaluate the performance of several turbulent algebraic stress models. 10.1.1: They compare the numerical results with LDV measurements in a free-surface turbulent jet. All the models assume a tlat free surface (i.c. zero Froude number). They find significant discrepancies between the predicted and measured flow behavior. Computational tools for free-surface turbulent shear flows are at an early stage of development.

Previous work on free-surface jets ${ }^{2.3 .5}$ has considered the steady jet in still water. Here a pulsed jet in coflow is used. This more complicated flow is studied because it captures more accurately the turbulent flow in the near field of a ship wake. The jet is pulsed to provide a phase reference for time resolved measurements of the large scale turbulent motions. The coflow also eliminates recirculation in the test facility and provides a vehicle for seeding the ambient fluid when using particle techniques.

There have been several investigations of both pulsed deep jets and deep jets with a coflow. Crow and Champagne "studied the response of a turbulent jet to forcing of varying frequency and amplitude. They concluded that the preferred mode corresponds to a Stroubal number of 0.30 and that an RMS forcing amplitude of $2 \%$ of the mean how is enough to saturate this mode four diameters downstream from the nozzle. Above a Reynolds number of about 10,000 they observed the large scale structure of the jet to be made up of vortex "puffs" forming at a Strouhal number of 0.3 .

Browand and Laufer ${ }^{1+}$ also report on their study of large scale structures in the development of a circular jet in the Reynolds number range 5,000 to 15,000 . They describe three regions of flow development: an initial shear layer instability zone, large scale vortex interaction region along the potential core $(x / d<4)$. and finally a zone of higher order turbulence generation. They report vortex passage in the very near field $(x / d<3$ say) at frequencies corresponding to Strouhal numbers near 1.0 (these vortices are associated with the initial laminar instability) but the passage rate quickly decreases to a steady state Strouhal number of about 0.5 at $x / d=4$. This Strouhal number is larger than the 0.3 found by Crow and Champagne.

Zaman and Hussain ${ }^{15.16}$ studied the large scale structure in the near field of an axisymmetric jet with and without controlled periodic forcing. A phase-averaged hot-film technique was used to determine the vorticity distribution of the large scale structures. They show that the naturally occurring large scale structures agree well with those induced by controlled excitation at low excitation level. Iligh levels of excitation produce considerably stronger structures with higher values of vorticity.

Liepmann and Gharib ${ }^{17}$ show the existence of streamwise vortices in the near field of the axisymmetric jet. They show these structures to be significant in the entrainment process and to dominate the entrainment process downstream of the potential core (presumably where axisymmetric vortical structures begin to break down).

The effect of a coflowing stream on the evolution of a round jet was investigated by Maczynski ${ }^{1 \times}$. He finds that the centerline velocity decays more slowly than in the free jec. Antonia and Bilger" studied experimentally an axisymmetric jet of air in a coflowing stream. Self preservation was not found for this flow and the behavior far downstream depends strongly on the complete flow history. In contrast, Bradbury and Riley" study the spread of a plane turbulent jet in a collowing stream. They found that simple dimensional arguments can be used to collapse the results indicating that the dynamics of planar and round jets are significantly different. Rajaratnam ${ }^{21}$ provides a comprehensive review of deep jets in stationary surroundings and in a coflowing stream.

In this work, the combined effects of collow and pulsing on the development of a free-surface jet are investigated. One objective is 10 clarify the role of the large scale, near-surface vorticity in the dynamics of the flow. To accomplish this it is necessary to measure the instantaneous subsurface velocity field with adequate temporal resolution. In this way the temporal evolution of the large scale structures may be found. A second objective is to determine the mean flow behavior of the turbulent free-surface jet in a coflowing stream. This includes the scaling of the surface current, jet growth rate, and centerline velocity decay rates and how these are effected by jet pulsing and coflow. It is part of this objective to explore the use of ensemble averaged PIV data to acquire spatially resolved statistical flow quantities as well as the instantaneous velocity data.

\section{Flow FaCILITY AND INSTRUMENTATION}

The jet flow experiments were conducted in a $2^{\prime} \times 2^{\prime}$ water channel. The channel was specially designed and built for this work and has unique features to accommodate advaneed optical diagnostics. Figure 2 shows a sketch of the water channel. The rough dimensions are 28 feet long by nine feet wide by nine feet high. The test section is two feet by two feet by eight feet long and the channel holds about 5,000 gallons of water. When looking at the side view of Figure 2, water circulates counterclockwise, driven by a propeller pump located in the lower circular pipe section. The pump is driven by a $5 \mathrm{HP}$ frequency-controlled electric motor and has a maximum volume flow rate of about 130 gallons per second. This pump provides test section velocities continuously variable from $4 \mathrm{~cm} / \mathrm{s}$ to about $130 \mathrm{~cm} / \mathrm{s}$. There is also an overhead truss mounted on top of the channel which supports a hoist system for installing and removing channel components and test apparatus. The hoist 


\section{Copyright (C)1997, American Institute of Aeronautics and Astronautics, Inc.}

system is not shown in rigure 2. The top of the channel is uncovered so that the surface is free everywhere.

The jet flow for these experiments is axisymmetric with nearly uniform exit velocity (top-hat profile). This flow is generated by pumping water through a submerged pod which houses a settling chamber and a contracting nozzle. For some of the measurements, the jet tlow was forced in its preferred mode, with Strouhal number $\mathrm{St}=\mathrm{fd} / \mathrm{U}=0.3$. $^{1 .}$ Forcing was implemented to organize the large scale turbulent structures and provide a phase reference for time resolved measurements. Figure 3 shows a sketch of the jet assembly. Two struts support the jet pod and allow positioning of the jet at the desired depth. The struts are connected to a base plate which is, in turn, mounted to the top of the test section. Water is fed to the jet pod through a vertical pipe. At the base of this pipe, the flow is turned 90 degrees and expanded into the settling chamber, after which the flow is contracted and leaves the jet pod as a uniform stream. The unforced turbulence level at the jet exit is about $2 \%$. The design is based on the nozzle design used by Anthony."

Figure 3 shows the complete jet tlow assembly. Water is supplied by a centrifugal pump from the water channel itself and some of the water may be returned to the channel to reduce the net pressure rise. Several valves control the overall flow rate and the fraction of the flow going through the rotating valve. A flowmeter is used to measure the average flow rate to the jet. Forcing of the jet is accomplished by allowing some of the supply water to pass through a rotating valve. The valve is driven through a speed reduction timing belt by a DC motor with feedback speed controller. This systern provides a valve rotational velocity uniform to better than $1 \%$ over a wide speed range. Phase reference is provided by an optical pickup located on the valve drive pulley.

The tlow was characterized using several measurement techniques namely: Planar PIV. Holographic PIV and hot film anemometry. Only the PIV system is briefly described here. Additional details on the PIV system and the other measurement techniques can be found in Reference 23. Planar PIV was used 10 measure instantaneous flow fields that are phase referenced to the pulsed jet, and also to obtain data ensembles for calculation of statistical quantities for a variety of tlow conditions. The PIV system was configured as follows. Particles were illuminated by a doubly pulsed laser sheet and their side-scattered images recorded on $35 \mathrm{~mm}$ photographic film (Kodak TMAX 400). Image analysis was done using a Young's fringe technique on a system described by Kwon."

Both vertical and horizontal light sheets were used to record PIV images in the free surface jet flow. In both orientations, the sheets were parallel to the jet centerline. A schematic of the conliguration used to record the horizontal images is shown in Figure 4. Both a top view and an end view of the water channel test section are shown along with the PIV recording hardware. The light source is a pair of Nd:YAG lasers coupled together so that the beams are collinear and can be pulsed independently to provide an arbitrary pulse separation. The outputs are frequency doubled by a harmonic generator into the green $(532 \mathrm{~nm})$. Mirrors direct the beam to the sheet forming optics, consisting of a spherical lens (1000 mm f.I.) and a concave cylindrical lens (25 $\mathrm{mm}$ f.l.) which together form a thin sheet, with a waist thickness of about $150 \mu \mathrm{m}, "$ in the water channel test section. The sheet forming optics are mounted on an optical rail and can be traversed up and down to position the light sheet vertically in the test section.

Seeding of the flow was done with $6.44 \mu \mathrm{m}$ plastic particles (Bangs Laboratories) which have a specific gravity of 1.19. These particles were uniformly dispersed throughout the water channel at a concentration of about $10,000\left(\mathrm{cc}^{\prime}\right)$ to achieve the recommended ten particles per interrogation volume at the magnifications used here. ${ }^{26}$ These particles follow the flow well and produce adequate scattering for image recording.

Particle images were recorded using a $35 \mathrm{~mm}$ SLR camera (Nikon FM2) with a $135 \mathrm{~mm}$ lens (Nikon) at $f 2.8$. An autowinder (Nikon MD-12) was used to electronically trigger the shutter and to provide rapid framing for multiple recordings. The position of the camera can be seen in Figure 4. It is placed below and to the side of the test section and views the light sheet through a nearby small rotating mirror and a large mirror which is located directly below the test section. The camera can be moved relative to the large mirror to change the magnification. Film to flow magnifications from 1:10 to $1: 5$ are obtainable and values of $1: 10$ and 1:6.7 were used for the present work.

To remove directional ambiguity, and to provide control over the particle image displacement range, a velocity bias system was used. The bias is achieved by shifting the second image of a pair relative to the first with a rotating mirror. ${ }^{37}$ In this case, a small oscillating (scanning) mirror (General Scanning model G325DT) is placed directly in front of the camera lens (see Figure 4 ) so that the line of sight from the camera to the flow field is turned approximately 90 degrees by the mirror. The mirror is driven by a General Scanning CX660 scanner controller which oscillates it in a sinusoidal manner. The image acquisition system (camera and laser flashlamps) is synchronized with the scanner so that images are always recorded when the mirror is at the maximum rotation rate. The combination of mirror oscillation amplitude and frequency in conjunction with the magnification of the system produce the desired bias velocity. In all cases discussed here the oscillation amplitude was quite small, about a fraction of a degree.

The PIV recording system is electronically synchronized to provide automation and repeatability. The system was operated in two modes, one for mean flow measurements and the other for measurements phase-locked to the pulsed jet. In both modes, a master signal is used to synchronize the lasers, scanning mirror, and camera.

The Planar PIV data is used to resolve turbulent structures in time and space by phase-locking the PIV recordings to the pulsed jet. The pulsing is used to organize the large scale structure and provide a phase reference for time resolved measurements. Twocomponent velocitics were measured in selected planes of interest at specific phases of the pulse period, allowing calculation of vorticity and strain fields and analysis of the evolution of large scale structures in the near ficld. Four phases are considered, $\Phi=$ $0^{\circ}, 90^{\circ}, 180^{\circ}, 270^{\circ}$ where a phase of zero degrees coincides with the fully open position of a rotating valve used to pulse the flow. For these experiments, $U_{c}=50 \mathrm{~cm} / \mathrm{s}$, and $U_{c}=5 \mathrm{~cm} / \mathrm{s}$ and the jet is pulsed at a frequency of $6 \mathrm{~Hz}$, corresponding to its preferred 
mode $(S t=0.3)$. These pulsed-jet conditions are repeated in mean flow measurements to determine the effect of pulsing on the mean flow development.

Mean and turbulence data were obtained from using the PIV system. Only a relatively small number of images were used to compute the mean flow properties. In order to determine the accuracy of the measurement, as well as the bias velocity, zero flow velocity images were processed. Measurements were made at the same points used in the flow cases. The results show a measurement uncertainty is $2 \%$ of the maximum velocity (bias plus flow) for both components of velocity. It corresponds to about $4 \%$ of the maximum excess velocity for both velocity components. The uncertainty of the RMS fluctuations is larger. The $75 \%$ confidence interval is $+25 \%-15 \%$ of the measured value.

\section{RESULTS}

\section{Scaling of Free Surface Jet in a Coflow}

Both hot-film and PIV data were obtained along linear paths, across the jet, at several downstream locations. These data were statistically analyzed to provide mean flow and turbulence quantities. A schematic of representative streamwise velocity profiles indicating the relevant jet parameters in the current notation is shown in Figure 5. Here $d$ is the jet diameter, $U_{e}$ is the jet exit velocity (for a top-hat profile), $U_{c}$ is the coflow velocity, $U_{m}$ is the mean maximum streamwise velocity at a given downstream location, and $U$ is the local mean streamwise velocity component. The parameter $\mathrm{L}_{\mathrm{y}}$ is the distance from the centerline to the half-excess-velocity point in the profile. Following the notation of Rajaratnam ${ }^{21}$ the parameter $\alpha$ is defined to be the ratio of exit to conow velocity $\left(\alpha=\mathrm{U}_{\mathrm{e}} / \mathrm{U}_{\mathrm{c}}\right)$.

Similarity of the horizontal mean velocity profiles occurs very early as can be seen in Figures 6 and 7 . In these figures, normalized excess velocity is plotted versus horizontal distance normalized by the local jet half-width, $L_{y}$. The two data sets were obtained with hot-film anemometry for the flow condition $U_{s}=$ $50 \mathrm{~cm} / \mathrm{s}, U_{c}=5 \mathrm{~cm} / \mathrm{s}$. One case is steady flow, the other is with the jet pulsing at $u_{e} / U_{e}=5 \%$. Part (a) of each figure shows the profiles at the jet centerline and part (b) shows protiles at the surface. Even as close as $x / d=4$, the profiles exhibit similarity for both cases. Clearly these profiles can be described by an equation of the form,

$$
\frac{U-U_{c}}{U_{m}-U_{c}}=f(\eta) ; \quad \eta=\frac{y}{L_{y}} .
$$

\section{Centerline Velocity Decay}

One important measure of the development of a jet is the decay of the centerline velocity. In the current work a varicty of thow conditions are considered which will effect the behavior of the jet as manifested by the centerline velocity decay. In order to properly characterize these effects the relevant velocity and length scale should be identify. The proper velocity scale can be derived from momentum conservation (sec for example Tennckes and Lumley". Rajaratnam ${ }^{21}$ ). The momentum flux integral for the axisymmetric jet in coflow can be written:

$$
M=\int_{0}^{\infty} 2 \pi \rho U\left(U-U_{c}\right) r d r,
$$

where $r=d / 2$ is the radius of the jet nozzle, and $\rho$ is the density. At the jet exit, the momentum tlux reduces to

$$
\mathrm{M}_{0}=\frac{\pi \mathrm{d}^{2}}{4} \rho \mathrm{U}_{\mathrm{e}}\left(\mathrm{U}_{\mathrm{e}}-\mathrm{U}_{\mathrm{c}}\right)=\text { constant }
$$

The mean excess velocity profiles are self-similar and can be written as a function of the similarity coordinate, $\eta$, as

$$
\frac{U-U_{c}}{U_{m}-U_{c}}=f(\eta) ; \quad \eta=r / L(x) \text {. }
$$

Substituting this expression for $\mathrm{U}$ in the momentum flux integral gives

$$
\begin{gathered}
M=2 \pi \rho \int_{0}^{\infty} L^{2}(x)\left[\left(U_{m}-U_{c}\right) f(\eta)+U_{c}\right]\left(U_{m}-U_{c}\right) f(\eta) \eta d \eta \\
M=2 \pi \rho L^{2}(x)\left(U_{m}-U_{c}\right) \\
{\left[\left(U_{m}-U_{c}\right) \int_{0}^{\infty} t^{2}(\eta) \eta d \eta+U_{c} \int_{0}^{\infty} f(\eta) \eta d \eta\right]} \\
M=2 \pi \rho L^{2}(x)\left(U_{m}-U_{c}\right)\left[\left(U_{m}-U_{c}\right) l_{2}+U_{c} l_{l}\right]
\end{gathered}
$$

where

$$
\begin{aligned}
& I_{1}=\int_{0}^{\infty} f(\eta) \eta d \eta=\text { const. } O(1) \\
& I_{2}=\int_{0}^{\infty} \mathrm{f}^{2}(\eta) \eta \mathrm{d} \eta=\text { const. } O(1) .
\end{aligned}
$$

The momentum flux is, therefore, the sum of two terms, one a contribution from the momentum in the excess velocity part of the profile, and the other a contribution from the coflow. These two terms contribute to varying degrees depending on the flow regime. Near to the jet exit, in the jet-like limit, the excess velocity term dominates (i.e. $\left(U_{m}-U_{c}\right) \gg U_{c}$ ), but far from the jet exit, in the wake-like limit, the collow term dominates. The operating regime then dictates the dominant term and therefore the choice of velocity scale.

In the present work, the main emphasis is on the near-field of the jet and thus the excess velocity is chosen as the velocity scale. It will be seen that the flow conditions considered here include the regime where the two contributions to the momentum flux are important, but that this regime is entered from the jet-like side. Antonia and Bilger, ${ }^{14}$ and Rajaratnam ${ }^{21}$ have used the coflow velocity, $U_{c}$, as the velocity scale but were primarily concerned with the wake-like regime. Use of the coflow velocity as a velocity scale breaks down as the coflow goes to zero, which is undesirable for comparison of the present results to noncoflowing jets.

The relevant length seale is derived from dimensional considerations. For the deep cotlowing jet, 


$$
U_{m}-U_{c}=f\left(M_{0}, \rho, x, U_{e}, U_{c}\right)
$$

thus the excess velocity ratio is a function of two nondimensional parameters

$$
\frac{U_{m}-U_{c}}{U_{c}-U_{c}}=f\left(\frac{U_{e}-U_{c}}{\sqrt{\frac{4 M_{0}}{\pi \rho}}} \times \frac{U_{e}}{U_{c}}\right) .
$$

The factor $4 / \pi$ in the first parameter arises when the initial momentum flux, $M_{0}$, is written as a function of a momentum thickness based on the exit excess velocity

$$
M_{0}=\frac{\pi d^{2}}{4} \rho U_{e}\left(U_{e}-U_{c}\right)=\frac{\pi \theta^{2}}{4} p\left(U_{e}-U_{c}\right)^{2}
$$

It follows that the centerline velocity decay scales with $x / \theta$. where $\theta$ is given by

$$
\theta=d \sqrt{\frac{U_{e}}{U_{e}-U_{c}}}=d \sqrt{\frac{\alpha}{\alpha-1}} .
$$

The above analysis assumes that at the jet exit, both the jet velocity and the collow velocity are uniform as sketched in Figure 5. The coflow stream however experiences interference from the presence of the pod which houses the jet. The effect of the pod wake can be characterized in terms of the wake momentum loss, $M_{w}$, given by

$$
M_{W}=\int_{\lambda} \rho U\left(U_{L}-U\right) d A
$$

where $A$ is the cross-sectional area, at $x / d=4$. This integral was evaluated using the measured data. The corresponding drag coefficient based on pod cross-sectional area $\left(\pi d_{p}^{2} / 4\right.$, where $d_{p}=$ $8.3 \mathrm{~cm}$ is the jet-pod diameter), was found to be

$$
C_{d}=\frac{M_{w}}{\frac{1}{2} \rho U_{c}^{2} \frac{d_{p}^{2}}{4}}=0.19 .
$$

The drag from the jet pod has the most pronounced effect when $\alpha$ is small. Of the cases studied herc, the jet-flow case for $\alpha=2.5$ where $U \mathrm{c}=50 \mathrm{~cm} / \mathrm{s}$, and $U \mathrm{c}=20 \mathrm{~cm} / \mathrm{s}$ has an excess velocity momentum flux of $M_{0}=0.076 \mathrm{~N}$. The wake effect is characterized in terms of the momentum flux ratio, $\beta$, which for this case is

$$
\beta={ }^{M} \mathrm{w} / \mathrm{M}_{0}=0.26
$$

A general relation for $\beta$ can be obtained as

$$
\begin{aligned}
& \beta=\frac{M_{W}}{M_{0}}=\frac{1}{2} C_{D}\left(\frac{d_{p}}{d}\right)^{2} \frac{U_{c}^{2}}{U_{c}\left(U_{c}-U_{c}\right)}= \\
& \frac{1}{2} C_{D}\left(\frac{d_{p}}{d}\right)^{2} \frac{1}{\alpha \cdot(\alpha-1)}=\frac{1.0}{\alpha(\alpha-1)} .
\end{aligned}
$$

From this relation, it is apparent that the momentum loss from the jet-pod wake will only be significant for small values of $\alpha$ (less than about four). For the present data set only the catse $\alpha=2.5$ satisfies this condition. The other tlow conditions consider in the present work have $\alpha$ equal to ten or twenty and so there should not be a significant wake effect $(\beta=0)$.

To account for the pod wake effect in the jet growth scaling, a new momentum thickness $\theta^{\prime}$ is defined

$$
M_{0}-M_{W}=\pi \theta^{\prime 2} \rho\left(U_{e}-U_{c}\right)^{2}
$$

and the ratio of the two momentum thickness gives

$$
\frac{\theta^{\prime}}{\theta}=\sqrt{1-\frac{M_{W}}{M_{0}}}=\sqrt{1-\beta}
$$

For the large collowing velocity case $(\alpha=2.5), \theta^{\prime} / \theta=0.86$. The ratios for the other cases are essentially equal to one.

Using this wake-corrected momentum thickness as the length scale, the centerline decay is plotted in Figure 8 . The present data exhibits an early increased decay rate for small $\alpha$, similar to Antonia \& Bilger," and then breaks to follow the free-surface jet data of Madnia \& Bernal atter the flow has significantly interacted with the surface $\left(x / \theta^{\prime}=12\right)$. The data of Walker et al. ${ }^{5}$ was obtained for a jet depth of $h / d=2.0$ and lies between that of Madnia and Bernal and the deep jet. This is attributed to the larger value of $h / d$ which results in a downstream shift of the interaction region. The present data extends to a maximum downstream distance of $x / \theta^{\prime}=16$, just entering the reduced decay rate region.

An interesting observation is the increased decay rate associated with increasing coflow. This effect is evident in the data of Antonia and Bilger and in the current case with large coflow velocity. It is a result of the change in thow regime between jet-like and wake-like behavior. In the equation for the conservation of momentum (equation (1)) there are two terms which contribute to the momentum flux. The first is dominant in the jet-like regime but as the jet decays the second one becomes significant as well. The result is that the quantity in brackets becomes increasingly larger, thus requiring the local excess velocity, $\left(U_{m}-U_{c}\right)$, to become smaller in order to conserve momenturn.

\section{Centerline Velocity at the Free Surface}

For a submerged jet, the surface flow velocity above the jet is initially very small. As the jet grows it interacts with the surface and induces motion there. Using the model of an image jet above the surface" which is valid at low Froude number, the surface can be viewed as a plane of symmetry and the maximum velocity will occur at the surface. Madnia and Bernal ${ }^{2}$ showed that the maximum surface velocity occurs at about $x / h=12$ and that the local maximum velocity in the profile is at the surface for $x / h>$ 30.

The centerline surface excess velocity is plotted versus downstream distance in Figure 9. The two plots contain data from two different depths below the surface; the first being 6.4 $\mathrm{mm}$ below the surface $(\mathrm{z} / \mathrm{h}=.75)$ and the second $2 \mathrm{~mm}$ below the 
surface $(z / h=.92)$. PIV data was obtained for all cases at the greater depth, but not all cases allowed measurement at the shallower depth due to large surface deformations for these cases (large Froude number and pulsing caused these effects). For one case $\left(U_{c}=50 \mathrm{~cm} / \mathrm{s}, U_{c}=5 \mathrm{~cm} / \mathrm{s}\right)$ the measurements were made 3 $\mathrm{mm}$ below the surface $(\mathrm{z} / \mathrm{h}=0.88)$ as this was the shallowest depth that did not intercept the deformed surface. Hot-film data was taken at $z / \mathrm{h}=0.92$ for two cases, one steaty and one pulsed. The data of Madnia and Bernal was also obtained at $z / h=0.92$ and is included in both plots for reference.

The near surface data agrees well with Madnia and Bernal's data, showing an initial rapid increase in surface velocity followed by a velocity decay, with the maximum surface velocity at $x / d=12$. At the greater depth $(z / h=0.75)$, closer to the jet centerline, the flow is accelerated sooner than at the surface and reaches a maximum at about $x / d=8$. The data of Madnia and Bernal is included for reference. When normalized in this manner, pulsing and coflow do not effect the streamwise surface velocity distribution although the hot-film data shows a slight increased velocity for pulsed flow.

\section{Jet Growth Rate}

The half width, $\mathrm{L}_{x}$, is plotted versus downstream distance normalized by $d$ in Figure 10(a). The data from the current investigation is plotted along with the data of Madnia and Bernal, and the coflowing data of Antonia and Bilger. The surtace je: data of Madinia and Bernal follows the deep jet closely but the cotlowing data diverges rapidly from the deep jet line and grows at a slower rate. The data from the present investigation follow that of Madnia and Bernal except for the large coflow case where the data breaks to follow the coflowing data. In Figure 10(b), the data has been plotted with the downstream distance normalized by $\theta^{\prime}$. This scaling brings the data more on line with the free jet, and corrects for the wake of the jet pod, but still shows the reduced rate of growth for large cotlow cases.

This decreased rate of growth for jets with large coflow is consistent with a change towards the wake-like regime discussed above. When $\alpha$ is small, this change happens quite carly since the decaying excess velucity and the coflow velocity quickly become of the same order. The effect of pulsing again is small with regard to the growth rate as it was in regard to the centerline velocity decay. The effect of coflow is dominant.

\section{Jet Spreading at the Surface - The Surface Current}

Anthony and Willmarth' found that near the surface there is a thin region of outward flow they termed the "surface current." They report that the surface current is first detectable at about $x / h$ $=8$ and is well established by $x / h=16$ where it was found to be about twice the width of the subsurface flow and growing at an included angle of about 80 degrees.

The data from the present experiments show the cxistence of a surface current. The greater lateral extent of the jet near the surface can be seen in the velocity profiles of the hot-film data. These effects are quantified in Figure 11 where the surfaco current half width is plotted versus downstream distance. Data at $7 / h=0.75$ and 0.92 are shown in separate plots. The half widths of the velocity profiles are slightly greater than the centerline width at $z / h=0.75$ but are significantly wider at $z / h=0.92$. The growth appears to be more rapid after $x / \theta^{\prime}=12$ which is consistent with the visualizations of Anthony and Willmarth'. The data does not show significant differences associated with pulsing or collow.

Another measure of the growth rate of the surface current is obtained from the instantaneous PIV velocily fields. This data was obtained at a several phases for the pulsed free-surface jet. The velocity fields were averaged to obtain a mean velocity field. In Figure 11, the contours of the mean streamwise velocity component are plotted for three cross-sectional planes aligned with the flow. Note that this is absolute velocity (U), not excess velocity $\left(U-U_{c}\right)$. Part (a) of the ligure is a vertical plane through the jet centerline. Parts (b) and (c) show horizontal planes at the centerline and near the surface $(z / h=0.75)$ respectively. A comparison of the horizontal planes at the centerline and near the surface reveals the formation of the surface current and its larger growth rate compared to the core of the jet. If the contour lines for $U=10 \mathrm{~cm} / \mathrm{s}$ are used as a measure of the extent of the flow, a quantitative comparison can be made. These contour lines diverge at an included angle of six degrees at the centerline plane, and at an angle of 22 degrees near the surface.

\section{Turbulence Structure}

Instantaneous velocity field measurements were made in a turbulent, pulsed, free-surface jet with coflow using Particle Image Velocimetry (PIV) and with the goal of resolving in space and time the large scale vortical structure. Turbulence statistics were also measured with a hot-film probe $\left(u^{\prime}\right)$ and with ensembles of PIV data ( $u^{\prime}, v^{\prime}, \overline{u v}$ ). This part of the discussion will concentrate on the instantaneous PIV data and will make use of the hot-film measurements and ensemble-averaged PIV dala for velocity fluctuation statistics.

\section{Instantaneous Structure}

PIV data was obtained in three image planes of the near ficld of the pulsed jet. The planes were all parallel to the jet axis, one vertical through the jet centerline, one horizontal through the jet centerline, and one horizontal near the free surface. Each image plane covered the full lateral extent of the flow and spanned from the exit plane through eight jet diameters downstream. The PIV recordings were phase locked to the jet pulsing frequency so that the organized structure in the jet could be recorded in the different planes for different realizations of the flow, but at the same phase. Figures 13 to 15 show velocily, vorticity, and strain rate fields, respectively, for the three planes, at a phase $\Phi=90^{\circ}$. Similar result were obtained for $\Phi=0^{\circ}, 180^{\circ}$ and $270^{\circ}$. The flow conditions for these experiments are: Average jet exit velocity, $U_{c}=50 \mathrm{~cm} / \mathrm{s}$, coflow velocity, $U_{c}=5 \mathrm{~cm} / \mathrm{s}$, jet exit diameter, $d=2.54 \mathrm{~cm}$, jet pulsing frequency, $f=6.0 \mathrm{~Hz}$, and jet centerline depth, $\mathrm{h} / \mathrm{d}=1.0$. Corresponding non-dimensional parameters are $\mathrm{Re}=12,700, \mathrm{Fr}=1.0$, and $\mathrm{St}=0.30$.

The forcing signal provides a phase reference for the large scale vortical structure in the flow. The calculated horizontal and vertical vorticity fields through the jet centerline show discreto vortex cores associated with circular vortices generated around the jet column. There are about six vortices along the length of the imaged regions implying an average spacing of about $30 \mathrm{~mm}$. Taking into account some reduction in mean how velucity along 


\section{Copyright (C)1997, American Institute of Aeronautics and Astronautics, Inc.}

this length, this spacing is about half that of the pulses, which is consistent with the expected propagation velocity of the vortices. As indicated earlier, the maximum magnitude of vorticity in these vortices $(\Omega / f=20)$ is in agreement with the results of other investigators. ${ }^{13}$ The vorticity levels decay to about $\Omega / f=7$ at $x / \mathrm{k}$ $=8$.

The present llow is unique from most previous sudies of urbulent structure in jets because of the presence of the free surface. The production and behavior of surface normal vorticity is one important aspect of the flow dynamics. The PIV data obtained in the horizontal plane close to the free surfice $(6.4 \mathrm{~mm}$ below, $t / \mathrm{h}=.75$ ) can be used to characterize the surface normal vorticity. The velocity, vorticity, and strain rate ficlds at the surface were measured tor pulse phases $0^{\circ}, 45^{\circ}, 90^{\circ}, 180^{\circ}, 135^{\circ}$ and $270^{\circ}$. A typical example is shown in Figure 13 to 15 . Surface normal vorticity is clearly present in these planes and appears in pairs indicating a horseshoe shaped reconnection of initially circular vortices with the free surface. The earliest appearance of these surface-normal vortices is at $\Phi=0^{\circ}$ where they are located about two jet depths downstrcam of the nozzle ( $x$ $=50 \mathrm{~mm}$ ). This is consistent with the finding of Madnia and Bcrnal that surface deformations first appear between onle and two jet depths downstream. If the progression of the location of the first vortex pair is followed from phase to phase, an approximate translational velocity is obtained at each phase. The average translational velocities, $U_{t}$, measured from these PIV data are: $\Phi=0^{\circ}$ to $90^{\circ}, U_{t}=72 \mathrm{~cm} / \mathrm{s} ; \Phi=90^{\circ}$ to $180^{\circ}, U_{t}=48$ $\mathrm{cm} / \mathrm{s} ; \Phi=180^{\circ}$ 10 $270^{\circ}, \mathrm{U}_{\mathrm{t}}=36 \mathrm{~cm} / \mathrm{s}$.

\section{Enstrophy Production at the Surface}

To characterize the effect of the free surface on the development of the vorticity near the free surface, the enstrophy transport equation will be used.

$$
\left[\frac{\partial}{\partial \mathrm{t}}+\underline{\mathrm{u}} \cdot \nabla-\frac{1}{\operatorname{Re}} \nabla^{2}\right] \frac{1}{2} \underline{\omega} \cdot \underline{\omega}=\underline{\underline{\varepsilon}}:(\underline{\omega} \underline{\omega})-\frac{1}{\operatorname{Re}} \nabla \underline{\omega}: \nabla \underline{\omega}
$$

where $\underline{\omega}$ is the vorticity vector and $\underline{\varepsilon}$ is the strain rate tensor. The left hand side of the equation gives the rate of change of enstrophy. The first term on the right hand side is $Q_{\omega}$ the enstrophy source term associated with vortex stretching. The second term on the right hand side is the enstrophy dissipation and is always negative. It is the enstrophy source term, $Q_{\omega}$, that is of interest here.

For low Froude number and a clean surface, the vorticity vector at the surface is normal to the surface since the shear stress goes to zero and there is no surface-normal velocity component. In this case, the vorticity and the strain rate tensor can be written as

$$
\underline{\omega}=\left(0,0, \omega_{z}\right), \text { and } \underset{\varepsilon}{=}=\left(\begin{array}{ccc}
\varepsilon_{x x} & \varepsilon_{x y} & 0 \\
\varepsilon_{y x} & \varepsilon_{y y} & 0 \\
0 & 0 & \varepsilon_{z z}
\end{array}\right)
$$

so that

$$
Q_{\omega}=\varepsilon_{z z} \cdot \omega_{z}^{2}
$$

Here it is seen that if there is positive strain (extensional stretching in the $z$ direction) then the enstrophy will be increased. Negative strain will reduce the enstrophy. The surfice normal strain can be determined using continuity

$$
\varepsilon_{x x}+\varepsilon_{y y}+\varepsilon_{z z}=0
$$

so that

$$
\varepsilon_{z z}=-\left(\varepsilon_{x x}+\varepsilon_{y y}\right)
$$

and

$$
Q_{\omega}=-\left(\varepsilon_{x x}+\varepsilon_{y y}\right)\left(\omega_{z}\right)^{2}
$$

The strain and vorticity can be calculated from the measured u and $v$ components of the velocity field in a horizontal plane near the free surface. The PIV data measured on planes parallel to the surface was used to determined $Q_{\omega}$ for the four pulse phases, $\Phi$ $=0^{\circ}, 90^{\circ}, 180^{\circ}$, and $270^{\circ}$. A typical contour plot of $Q_{\omega}$ is shown in Part (a) of Figure 16. The contour lines start at $\pm 5,000$ $\mathrm{s}^{-3}$ and are spaced every $5,000 \mathrm{~s}^{-3}$. Solid lines represent enstrophy production, dashed lines enstrophy reduction. If these contour plots are compared with their corresponding vorticity fields, the features of the enstrophy production ficld can be secn to correspond to those in the vorticity theld. This is expected because of the dependence of $Q_{\omega}$ on the vorticity squared. Perhaps the most striking feature of the data is the localized nature of enstrophy production at the surface.

Table 1.

Total Enstrophy Production, Total Enstrophy, and Production Rate

\begin{tabular}{|c|c|c|c|}
\hline$\Phi$ & $\frac{\mathrm{d} \sum\left(\mathrm{Q}_{\omega} \Delta x \Delta y\right)}{\left(U_{\mathrm{e}}-\mathrm{U}_{\mathrm{c}}\right)^{3}}$ & $\frac{\sum\left(\frac{1}{2} \omega^{2} \Delta x \Delta y\right)}{\left(U_{\mathrm{e}}-\mathrm{U}_{\mathrm{c}}\right)^{2}}$ & $\frac{\mathrm{d} \sum \mathrm{Q}_{\omega}}{\left(\mathrm{U}_{\mathrm{c}}-\mathrm{U}_{\mathrm{i}}\right) \sum \frac{1}{2} \omega}$ \\
\hline 0 & -0.65 & 4.45 & \\
\hline 90 & 0.41 & 4.09 & -0.15 \\
180 & -0.71 & 3.29 & -0.10 \\
270 & -0.15 & 3.38 & -0.05 \\
\hline
\end{tabular}

Also of interest is the total production of enstrophy at the free surface resulting from vortex stretching. The total production of enstrophy, along with the total enstrophy at the surface, and the associated fractional instantaneous increase/decrease in enstrophy are tabulated in Table 1 for the four pulse phases. The results are normalized using the jet exit excess velocity and diameter. Three of the four phases have a net decrease in enstrophy while the case $\Phi=90^{\circ}$ has an increase. For all cases the enstrophy production is quite large compared to the total enstrophy at a given instant.

The fact that the total enstrophy production is negative in most cases is interesting. The contour plots show islands of intense enstrophy production with areas and magnitudes at least as large as those in the regions of enstrophy reduction. There is a pattern 
of regions of large enstrophy production in the midst of a background of low level reduction. This is consistent with the formation of the surface current which requires net flow towards the surface and outward, creating a net compressional strain in the direction normal to the surface (i.e. negative $\varepsilon_{z z}$ ).

To gain a better understanding of the local enstrophy production, the streamwise variation of enstrophy production is considered. Part (b) of Figures 16 is a line plot showing the net value of $Q_{0}$ at each downstream location. The values were obtained by adding the local values of $Q_{\omega}$ dy over the range of $y$ values at each downstream location. For each phase, a large positive spike can be identified at the downstream location of the first reconnected vortex pair. this positive spike is immediately preceded by a negative spike. This behavior is indicative of a horseshoe shaped vortex oriented vertically across the flow with its open ends connected to the free surface. The lower, crosswise portion will induce a downward velocity in front, causing a positive surface normal strain, and hence extensional stretching of the vertically oriented vorticity. Behind the horseshoe vortex, the reverse happens, and the surface normal vorticity is compressed, resulting in enstrophy reduction there.

\section{$\underline{\text { Statistical Structure }}$}

Some lurther insight into the effect of pulsing on the structure of the turbulence may be obtained from the hot-film data. Two sets of measurements of the mean and fluctuating streamwise velocity component were made for the same mean flow conditions $\left(U_{e}=50 \mathrm{~cm} / \mathrm{s}, U_{c}=5 \mathrm{~cm} / \mathrm{s}\right)$ but in one case, the jet was pulsed in its preferred mode ( $\mathrm{St}=0.3$ ). This data is shown in Figures 17 and 18.

The most striking feature of this data is that the turbulence level is lower when the jet is pulsed. The exit turbulence level of the steady jet is about $2 \%$ of the exit excess velocity. At $x / d=4$, the turbulence level has increased to $30 \%$. In contrast, the exit turbulence level in the pulsed jet is $5 \%$ but at $x / d=4$, the turbulence level is only $20 \%$. Further downstream, the turbulence in the steady jet remains about $50 \%$ higher than in the pulsed jet. This finding is inconsistent with the results of Crow and Champagne ${ }^{13}$ who show increased turbulence levels in the pulsed jet up through $x / d=8$ at which point the levels become similar. They show peak turbulence ( $\left.u^{\prime} / U\right)$ in the forced case of approximately $18 \%$ at $x / d=4$ and, in the unforced case, about $14 \%$ at $x / d=8$.

One possible explanation for the loss in turbulent energy for the pulsed free-surface jet is that this energy is transferred to surface waves. Surface waves were observed to have much greater amplitude for the pulsed jet. Since these waves radiate from the region of surface interaction $(\sim x / d=4)$, turbulent energy will be removed from the subsurface flow. Walker et al." show a turbulence kinetic energy reduction of $20 \%$ in a jet with Froude number of 8.0 when compared to a jet with Froude number of 1.0. This linding is consistent with the above theory and implies greater wave production for the pulsed jet even at a lower Froude number.

\section{CONCLUSIONS}

An experimental investigation of a round, turbulent, freesurface jet has been conducted. The jet issued parallel to, and one jet diameter below the surface. The jet was operated in a collow of varying velocity and was, in some cases, forced in its preferred mode. Jet exit Reynolds numbers were 12,700 and 25.400 with corresponding Froude numbers of 1.0 and 2.0. The main conclusions of the investigation are summarized below.

- The mean flow behavior of the jet wass studied using velocity measurements along vertical and horizontal lines across the flow at various distances from the jet nozzle, as well as averaged planar velocity fields obtained with PIV.

- Streamwise mean velocity profiles became self-similar as close as four jet diameters downstream of the nozzle in horizontal planes both at the centerline and near the surface. This was true for both steady and pulsed jet flows.

$>$ The proper velocity scale in the near field of the coflowing jet is the exit excess velocity, $U_{e}-U_{\theta}$, and the proper length scale is the excess momentum thickness, based on exit excess velocity.

- The effect of a cotlow is to increase the decay rate in the initial region until interaction with the free surface slows the decaly rate as it does for non-cotlowing jets. The early, increased, decay rate of the coflowing jet is consistent with previous data and is interpreted here to be the result of the change from jet-like to wake-like behavior. This transition happens very early $(x / d<8)$ when the jet exit velocity to coflow velocity ratio is small $(\alpha=2.5)$

> The surface current was observed for all flow conditions. It results in an increased width of the horizontal mean velocity profiles near the surface relative to those at the jet centerline. At $\mathrm{x} / \mathrm{h}=16$, the width of the surface current is about twice that of the core of the jet. A similar conclusion was obtained from averaged PIV velocity fields in planes horizontal to the surtace which show that between $x / h=4$ and $x / h=8$. the spreading angle of the surface current is about $22^{\circ}$ compared to $6^{\circ}$ at the centerline. The measurce divergence angle of the surface current is consistent with a transition to a fully developed surface current with even greater divergence angle.

- The turbulence structure was analyzed using planar PIV data obtained in a vertical plane through the jet centerline, a horizontal plane through the jet centerline, and a horizontal plane near the surface. These instantaneous measurements were phase-locked to the pulsed jet so that the organized structure could be seen in the three planes at four phases of the pulse period. Turbulence statistics were obtained with a hot-film probe and with ensembles of PIV data.

- The large scale subsurface turbulence is shown to be made of generally ring-like structures produced at the pulsing frequency and propagating at about halt the local maximum velocity. This is consistent with the visualizations and measurements of Browand and Laufer $^{14}$ and others. 
- Surface normal vortex pairs tirst appear two jet depths downstream of the norzle. The translational velocity of the reconnected vortex pair is initially about $50 \%$ higher than that of the mean centerline flow but rapidly decays to a velocity about $50 \%$ below the mean centerline velocity and the two sides of the pair begin to move apart under the influence of the surface current. This occurs near the end of a pulse phase, at which point the next vortex reconnection appears.

- There is a net reduction in enstrophy at the free surface, consistent with the surface-normal compressive effects of the surface current, but there are local regions of intense production associated with the reconnected vortices. Ahead of the reconnected vortex cores, there is an enstrophy generation spike and behind the reconnected cores, there is a sharp reduction in enstrophy. This behavior is due to flow induced by the subsurface large scale vortical structures.

- The most significant effect of pulsing was found to be a reduction in turbulent velocity fluctuation as compared to the steady jet. This effect is attributed to increased surface wave generation for the pulsed jet which radiates turbulent kinetic energy away from the flow.

\section{ACKNOWLEDGMENTS}

This research was funded by the Fluid Dynamics program of the Office of Naval Research under Grant No. No0014-92-J1750, Dr. Edwin Rood program monitor. Their support is gratefully acknowledged.

\section{REFERENCES}

I Munk, W. H., Skully-Power, P., and Zachariasen, F. (1987) "Ships from Space," Proc. R. Soc. Lond. A, 412, 231-254.

? Madnia, C. K. and Bernal, L. P. (1994) "Interaction of a Turbulent Round Jet with the Free Surface," J. Fluid Mech., $261,305-332$.

3 Anthony, D. G. and Willmarth, W. W. (1992) "Turbulence measurements in a round jet beneath a free surface," J. Fluid Mech., 243, 699-720.

4 Swean, T. F., Ramberg, S. F., Plesnia, M. W. and Stewart, M. B. (1989) "Turbulent Surface Jet in Channel of Limited Depth," J. Hydraul. Eng., 115, 1587-1606

5 Walker, D. T., Chen, C.-Y., and Willmarth, W. W. (1995) "Turbulent Structure in Free-Surface Jet Flows," J. Fluid Mech, 291, 223-262.

6 Mangiavacchi, N. (1994) "Dynamics of a Turbulent Jet Interacting with a Free Surface," Ph.D. Thesis, University of Michigan

7 Gharib, M., Dabiri, D., and Zhang, X. (1994) "Interaction of small-scale turbulence with a free surface," In Free Surface Turbulence (ed. E. P. Rood \& J. Katz). ASME FED-181, p 97.

\& Walker, D.T., Leighton, R.I. and Garza-Rios, L.O. (1996) "Shear-frec turbulence near a flat free surface," J. Fluid Mech., 320, 19-51

"Walker, D.T. and Chen, C.-Y. (1996) "Evaluation of algebraic stress modeling in free surface jet tlows," J. Fluid Eng., 118 48-54.
10) Miner, E.W., Swean, T.F., and Troesh, A.W. (1988) "Evaluation an additional documentation of the parabolic marching code SURFWAKE," NRL Memorandum Report 6331, Naval Research Laboratory, Washington, D.C.

$"$ Swean, T.F., Leighton, R.I., Handler, R.A., Swearingen, J.D. (1991) "Turbulence modeling near the free surface in open channel flow," AIAA Paper 9/-0613.

12 Miner, E.W., Stewart, M.B. and Swean, T.F. (1993) "Modeling and computation of turbulent free-surface jets," AIAA Paper 93-0201.

${ }^{13}$ Crow, S. C. and Champagne, F. H. (1971) "Orderly structure in jet turbulence," J. Fluid Mech., 48, 547-591.

${ }^{14}$ Browand, F. K. and Laufer, J. (1975) "The Role of Large Scale Structures in the Initial Development of Circular Jets," Proc. 4th Symp. on Turbulence in Liquids, U of Mo.-Rolla, eds. J. L. Zakin and G. K. Patterson.

${ }^{15}$ Zaman. K. B. M. Q., and Hussain, A. K. M. F. (1981) "Taylor Hypothesis and Large-Scale Coherent Structures," J. Fluid Mech., 112, 379-396.

${ }^{16}$ Zaman, K. B. M. Q., and Hussain, A. K. M. F. (1984) "Natural Large-Scale Structures in the Axisymmetric Mixing Layer," J. Fluid Mech., 138, 325-351.

17 Liepmann, D. and Gharib, M. (1992) "The Role of Streamwise Vorticity in the Near-Field Entrainment of Round Jets," J. Fluid Mech., 245, 643-668.

Is Maczynski, J. F. J. (1962) "A Round Jet in an Ambient CoAxial Stream," J. Fluid Mech., 13, 597-608.

${ }^{19}$ Antonia, R. A. and Bilger, R. W. (1973). "An Experimental Investigation of an Axisymmetric Jet in a Co-Flowing Stream," J. Fluid Mech., 61(4), 805-822.

20 Bradbury. L. J. S. and Riley, J. (1967) "The Spread of a Turbulent Plane Jet Issuing Into a Parallel Moving Airstream," J. Fluid Mech., 27(2), 381-394.

${ }^{21}$ Rajaratnam, N. (1976) Turbulent Jets, Elsevier Scientific Publishing Company, Amsterdam.

22 Anthony, D. G. (1990) "The Intluence of a Free Surface on the Development of Turbulence in a Submerged Jet," Ph.D. Thesis, University of Michigan.

${ }^{23}$ Scherer, J.O. (1995) "Whole-Field Velocity Measurements in a Turbulent Frec-Surface Jet" Ph.D. Thesis, University of Michigan

${ }^{24}$ Kwon. J. T. (1988) "Experimental Study of a Vortex Ring Interaction with a Free Surface," Ph.D. Thesis, University of Michigan.

25 Shack, D. H., Bernal, L. P., and Shih, G. S. (1995) "Experimental Investigation of Underhood Flow in a Simplitied Automobile Geometry," Submitted for publication.

26 Adrian, R. J. (1991) "Particle Imaging Techniques for Experimental Fluid Mechanics," Ammu. Rev. Fluid Mech., 23, $26:-304$.

${ }^{27}$ Adrian, R. J. (1986) "Image Shifting Technique to Resolve Directional Ambiguity in Double-Pulsed Velocimetry," Appl. Opt., 25(21), 3855-58.

28 Tennekes, 11. and Lumley, J. L. (1972) A First Course in Turbulence, The MIT Press, Cambridge, Massachusetts. 
Copyright (c)1997, American Institute of Aeronautics and Astronautics, Inc.

29 Bernal, L. P. and Madnia, C. K. (1988) "Interaction of a

\section{FIGURES}

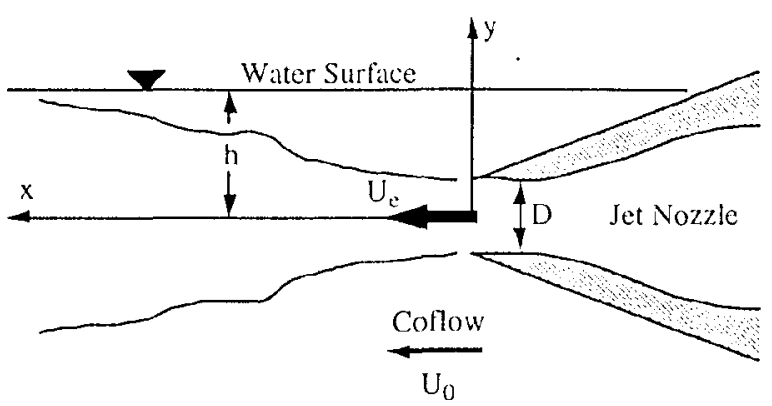

Figure 1. Schematic diagram of a free-surface jet.
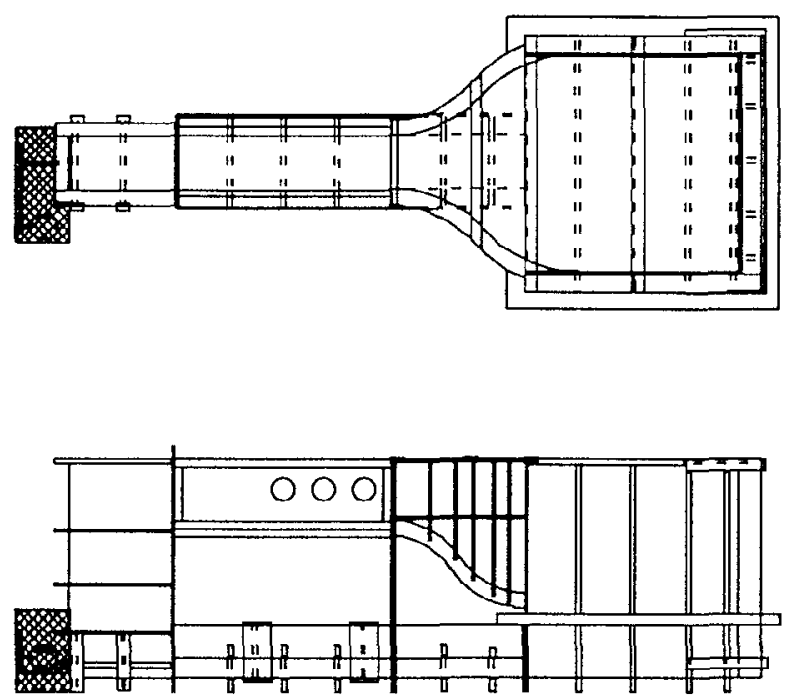

Figure 2. Sketch of 2' $\times 2^{\prime}$ Water Channel.
Turbulent Round Jet with the Free Surface," Proc. 17th Symp. Naval Hydro., The Hague, Netherlands.

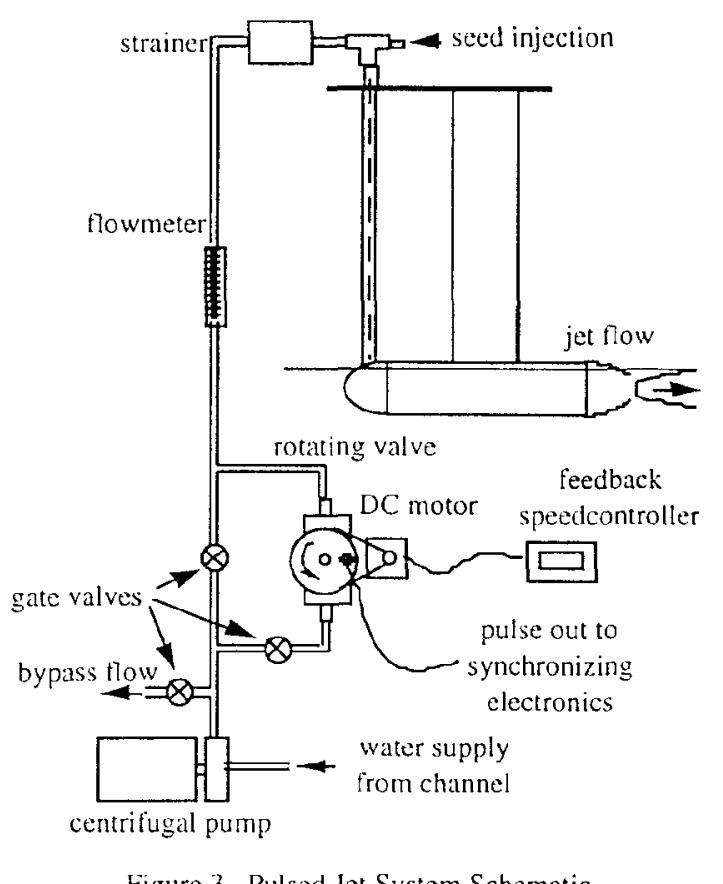

Figure 3. Pulsed Jet System Schematic 


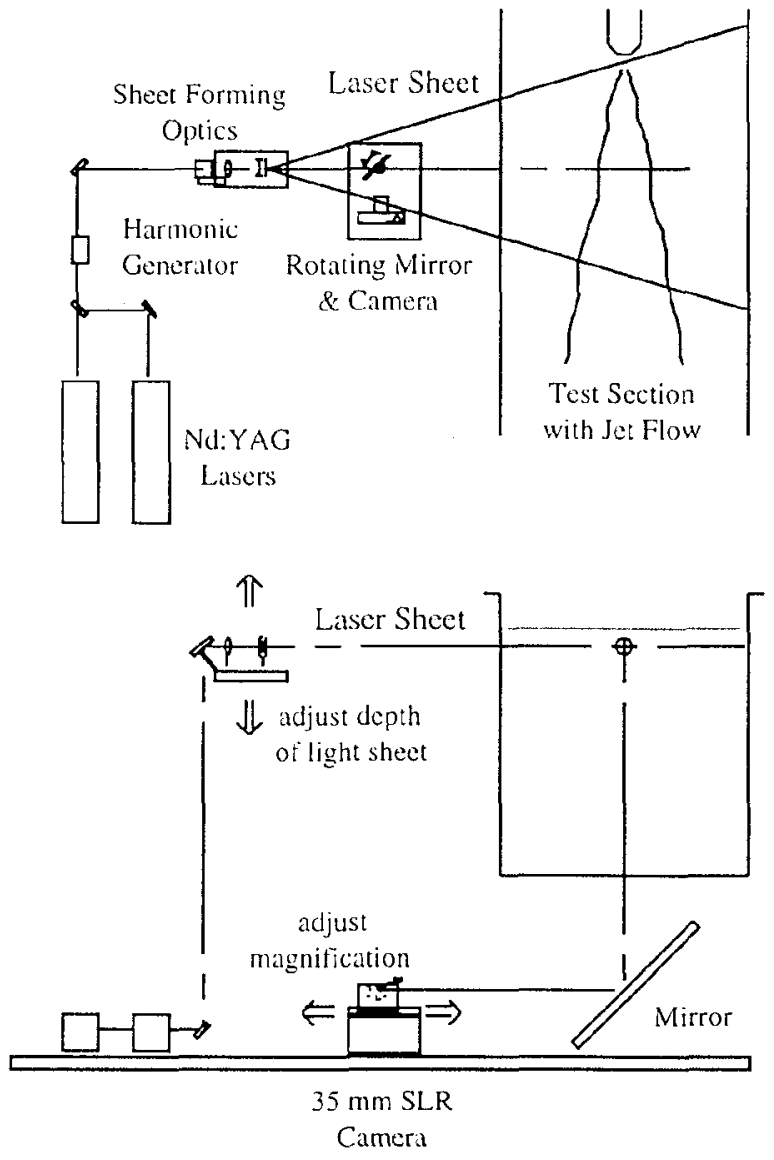

Figure 4. PIV Recording Configuration for Horizontal Planes.

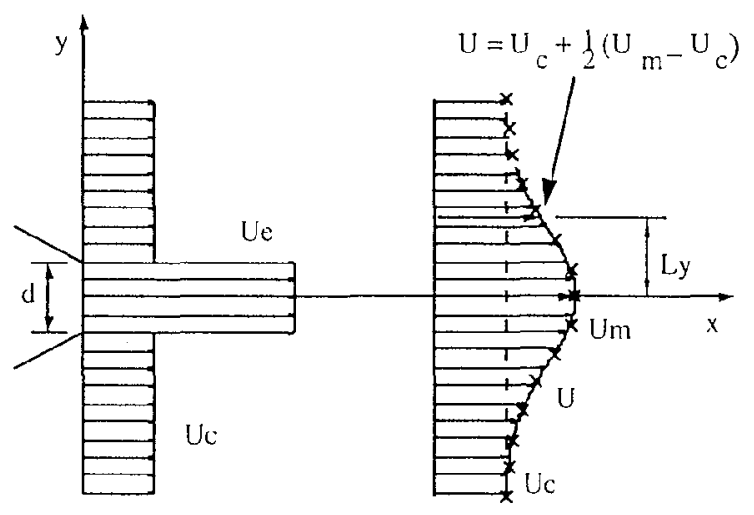

Figure 5. Schematic of Horizontal Velocity Profiles for Round Jet in a Collow

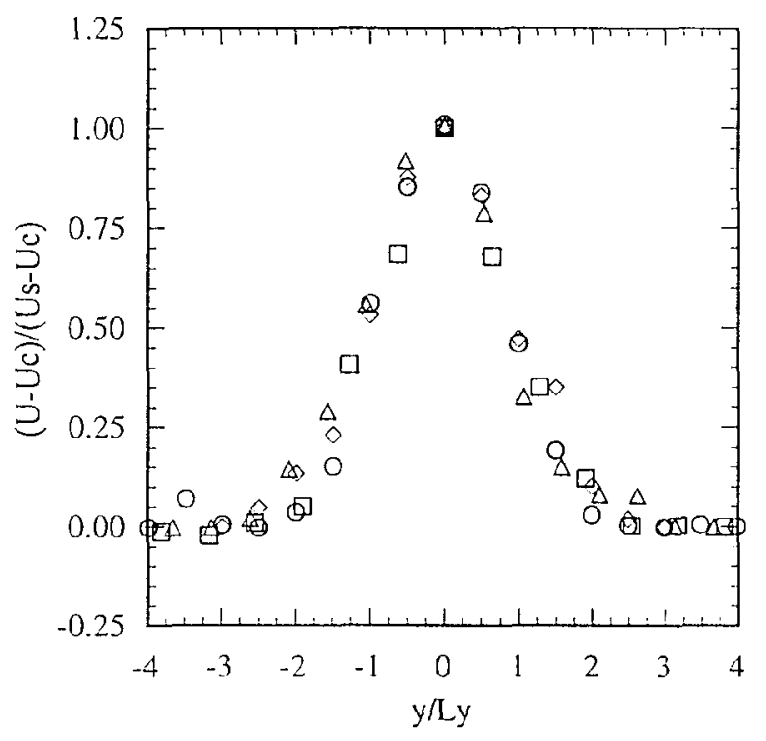

(a)

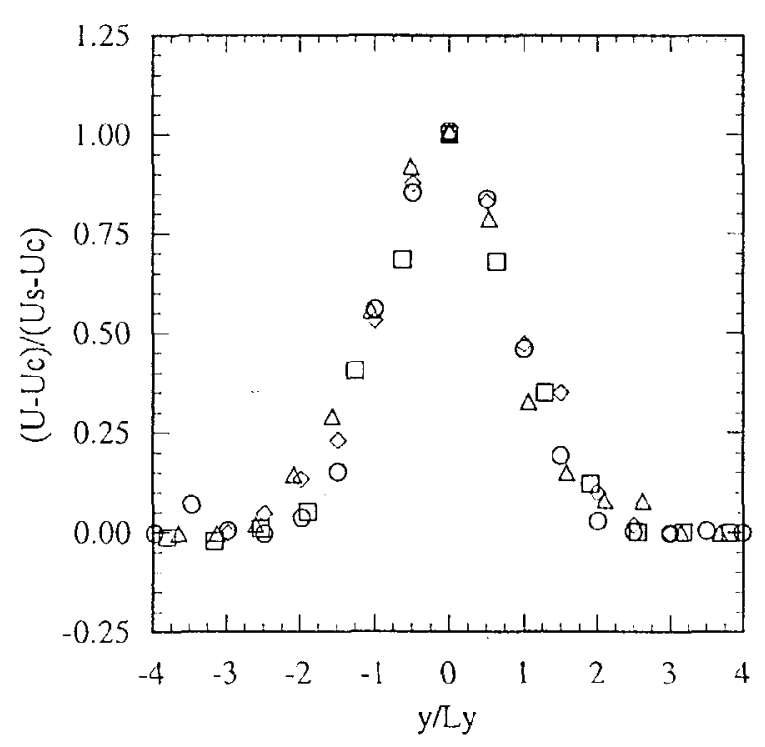

(b)

Figure 6. Horizontal Similarity Profiles for Baseline Stealy Flow, Taken from Hot-film Data. (a) Profiles through the jet centerline. (b) Profiles $2 \mathrm{~mm}$ below the surface $(z / \mathrm{h}=0.92)$. $\square, \mathrm{x} / \mathrm{d}=4, ; O, \mathrm{x} / \mathrm{d}=8 ; \Delta, \mathrm{x} / \mathrm{d}=12 ; 0, \mathrm{x} / \mathrm{d}=16$. 


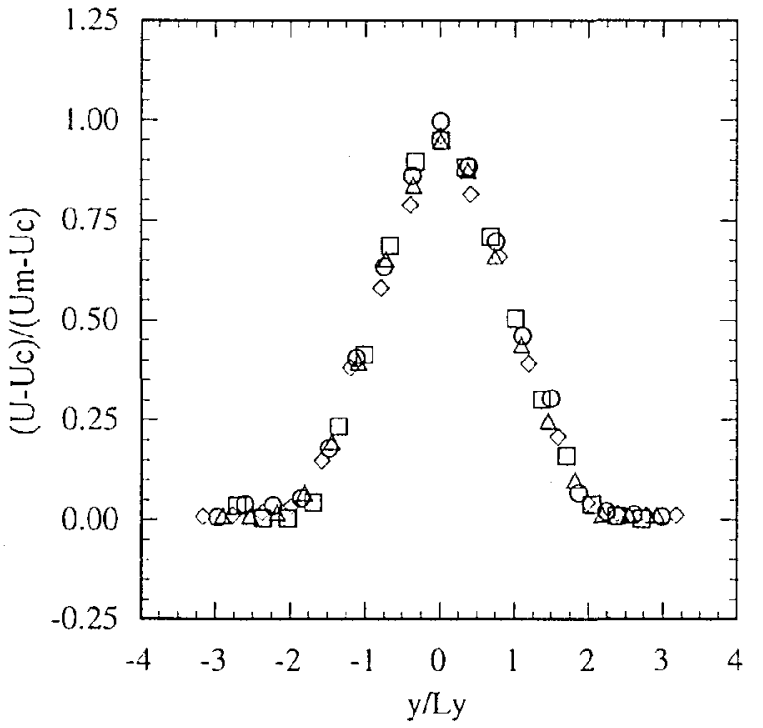

(a)

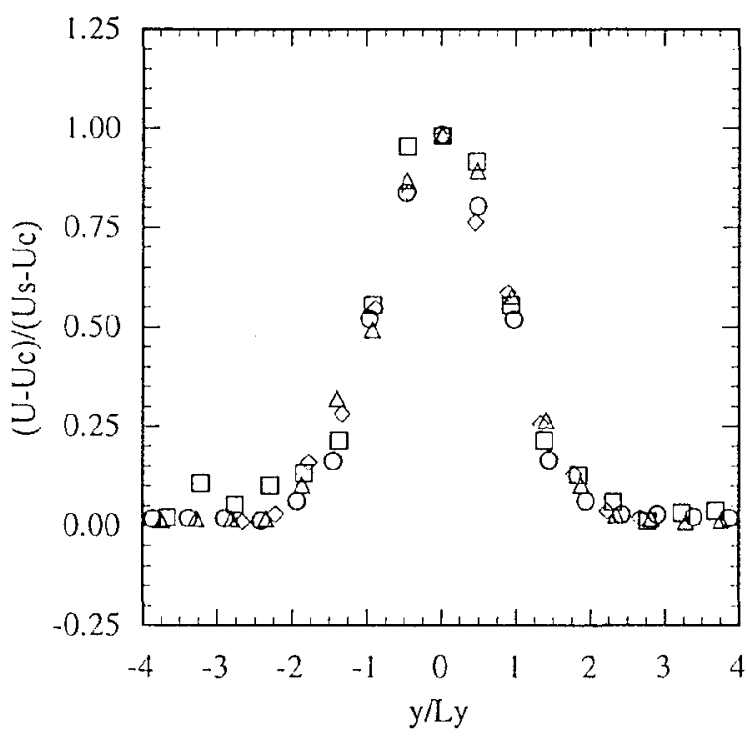

(b)

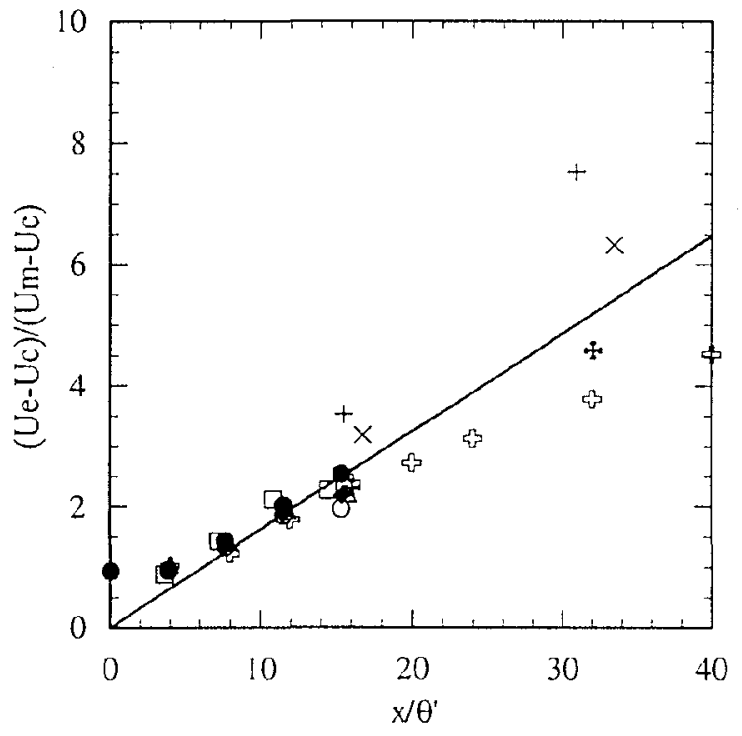

Figure 8. Centerline Excess Velocity Decay vs. Downstream Distance Normalized by Momentum Thickness. The data from the current investigation is plotted along with data from Madnia and Bernal, Walker, et. al., and Antonia and Bilger. Current data: - baseline case $(\alpha=10)$ - hot-film data; O, baseline case $(\alpha=10)$ - PIV data; $\bullet$, pulsed flow $(\alpha=10)$ - hot-film data; $\diamond$,pulsed flow $(\alpha=10)$ - PIV data; $\square$, large coflow $(\alpha=2.5)$; $\Delta$ small coflow $(\alpha=20)$. Other data: 3 . Madnia and Bernal $(\mathrm{h} / \mathrm{d}=1)$; +1. Walker, et. al. $(\mathrm{h} / \mathrm{d}=2) ;+$, Antonia and Bilger $(\alpha=3) ; X$. Antonia and Bilger $(\alpha=4.5)$. The solid line represents deep jet results.

Figure 7. Horizontal Similarity Profiles for Pulsed Flow, Taken from Hot-film Data. (a) Profiles through the jet centerline. (b) Protiles $2 \mathrm{~mm}$ below the surface $(2 / \mathrm{h}=0.92) . \square, \mathrm{x} / \mathrm{d}=$ $4 ; O, x / d=8 ; \Delta, x / d=12 ; \diamond, x / d=16$. 


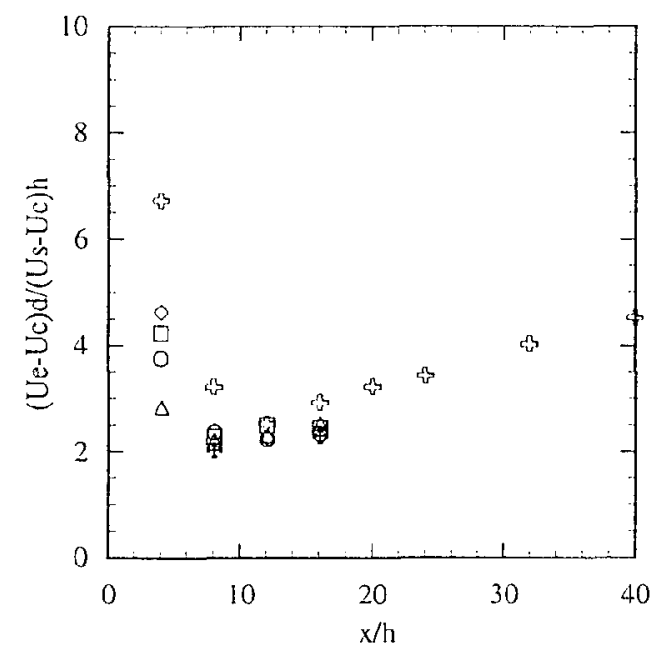

(a)

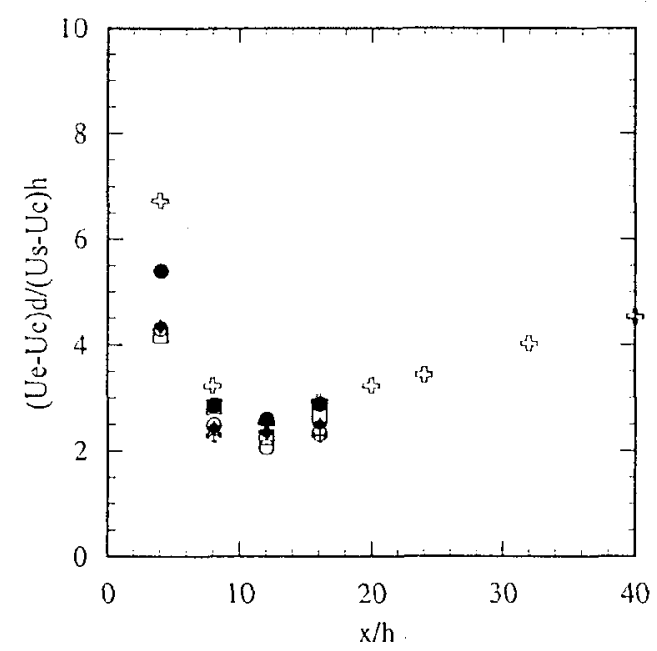

(b)

Figure 9. Centerline Excess Velocity at the Free Surface vs. Downstrcam Distance. The data has heen normalized by $h / d$ to permit comparison with the data of Walker, et. al. which was made at $h / d=2$ (other free-surface data is at $h / d=1$ ). (a) $6.4 \mathrm{~mm}$ below the surface $(z / \mathrm{h}=0.75)$, (b) $2 \mathrm{~mm}$ below the surface $(z / h=0.92)$. Current data: 0 , baseline case $(\alpha=$ 10) - hot-film data; $O$, baseline case $(\alpha=10)$ - PIV data: $\bullet$, pulsed flow $(\alpha=10)$ - hot-film data; $\diamond$, pulsed flow $(\alpha=10)$ - PIV data; $\square$, large collow $(\alpha=2.5) ; \Delta$ small collow $(\alpha=$ 20). Other data: $\{$. Madnia and Bernal $(h / d=1)$; $\Psi$, Walker et al. $(\mathrm{h} / \mathrm{d}=2)$.

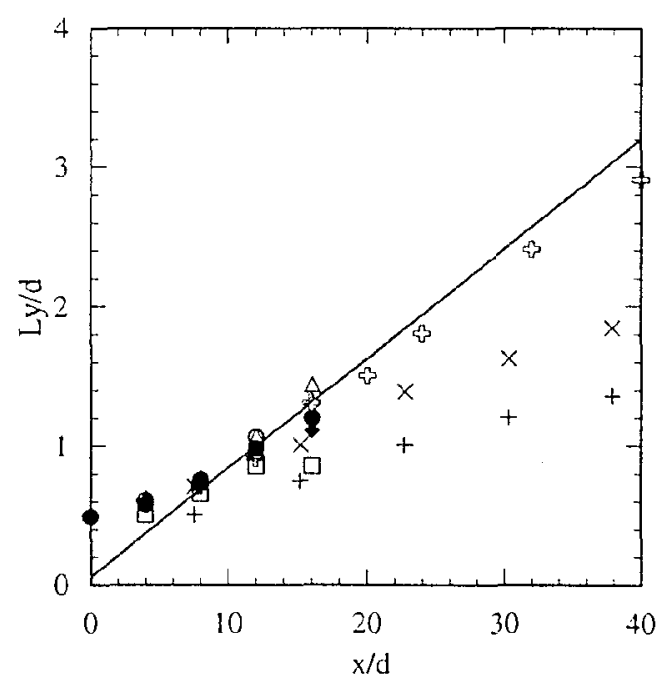

(a)

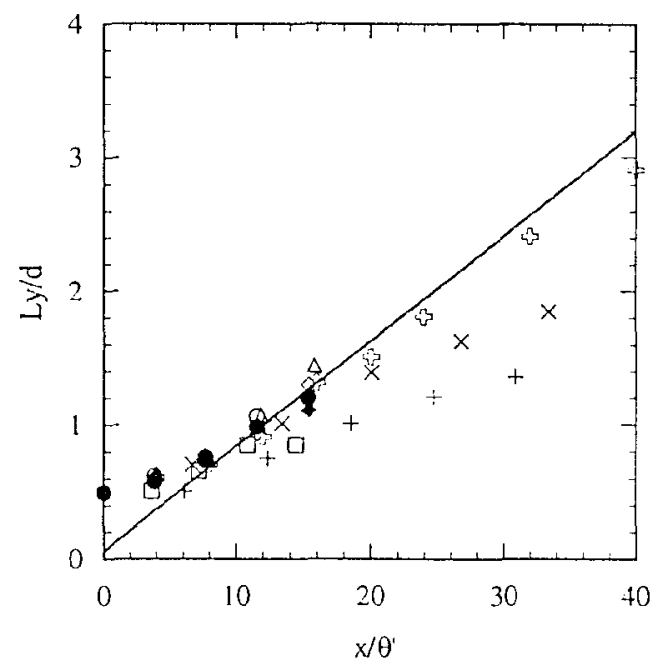

(b)

Figure 10. Horizontal Velocity Profile Half-Widths at the Centerline. The half-width $\left(\mathrm{L}_{\mathrm{y}}\right)$ is the horizontal distance from the jet centerline to the $y$ location where the local excess velocity is one-half the centerline excess velocity. The half-width is plotted vs. normalized downstream location ( $x$ ). In (a) $x$ is normalized by the jet diameter (d). In (b) by the jet exit excess momentum thickness $\theta^{\prime}$. Current data: 0 , baseline case $(\alpha=10)$ - hot-film data; $O$, baseline case $(\alpha=10)$ - PIV data; $\bullet$, pulsed flow $(\alpha=10)$ hot-film data; $\diamond$,pulsed flow $(\alpha=10)$ - PIV data; $\square$, large coflow $(\alpha=2.5) ; \Delta$ small coflow $(\alpha=20)$. Other data: $\xi^{\circ}$. Madnia and Bernal; +. Antonia and Bilger $(\alpha=3)$; $X$, Antonia and Bilger $(\alpha=4.5)$. The solid line represents deep jet growth. 


\section{Copyright (C)1997, American Institute of Aeronautics and Astronautics, Inc.}

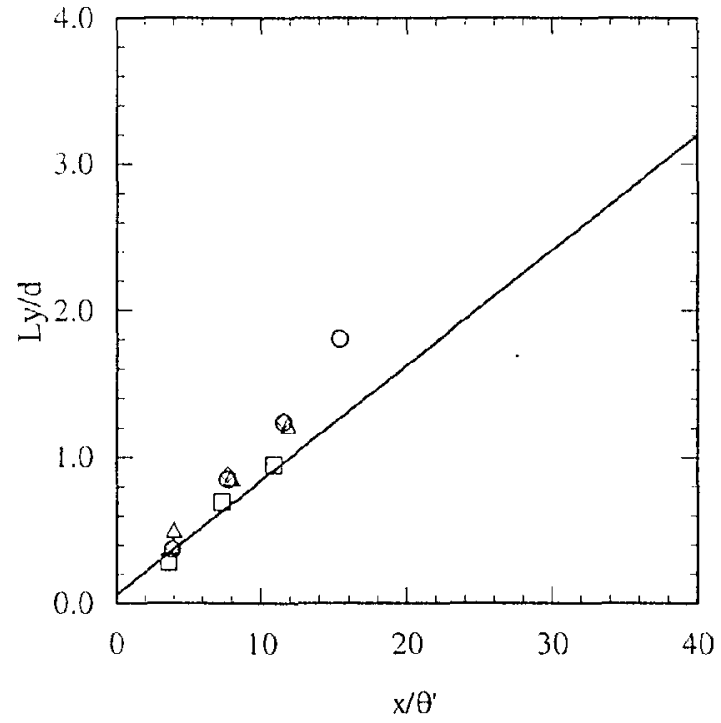

(a)

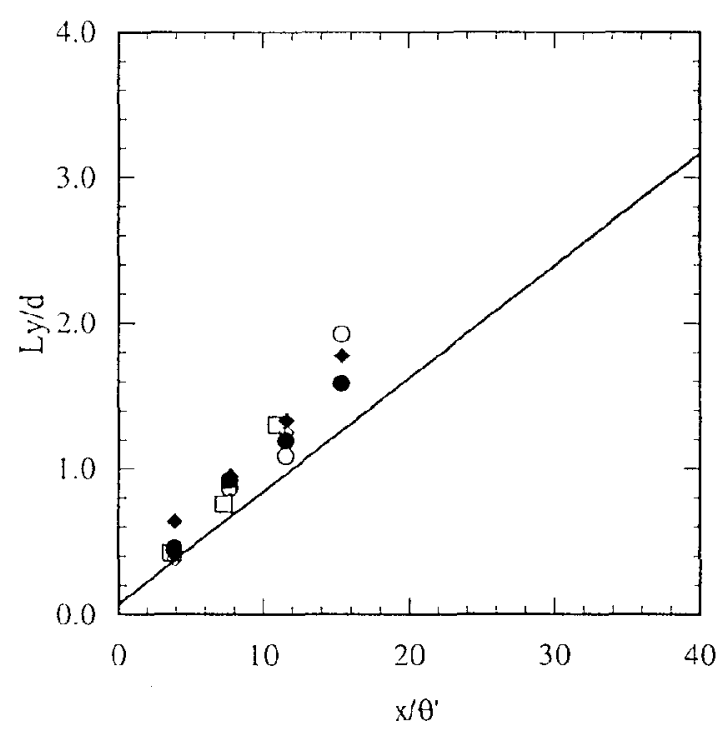

(b)

Figure 11. Horizontal Velocity Profile Half-Widths Near the Surface. The half-width $\left(L_{y}\right)$ is the horizontal distance from the jet centerline to the y location where the local excess velocity is onc-half the centerline excess velocity. The normalized halt-width is plotted vs. normalized downstream location $(x)$. In (a) the measurements are $6.4 \mathrm{~mm}$ below the surface $(z / \mathrm{h}=0.75)$, in (b) they are $2 \mathrm{~mm}$ below $(z / \mathrm{h})=$ 0.92. baseline case $(\alpha=10)$ - hot-film data; $O$, baseline case $(\alpha=10)$ - PIV data; $\$$, pulsed flow $(\alpha=10)$ - hot-film data; $\diamond$,pulsed flow $(\alpha=10)$ - PIV data; $\square$, farge coflow $(\alpha$ $=2.5): \Delta$ small coflow $(\alpha=20)$. The solid line represents deep jet growth.

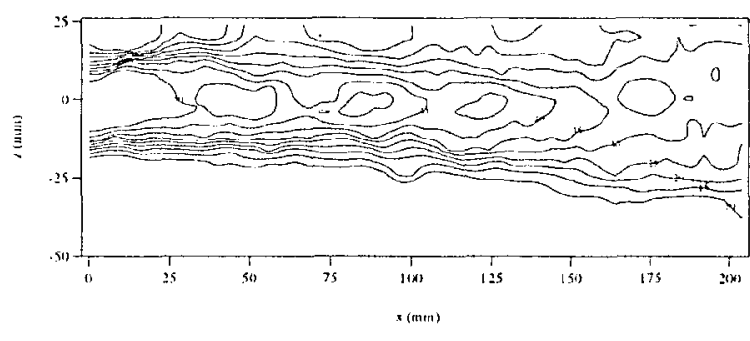

(a)

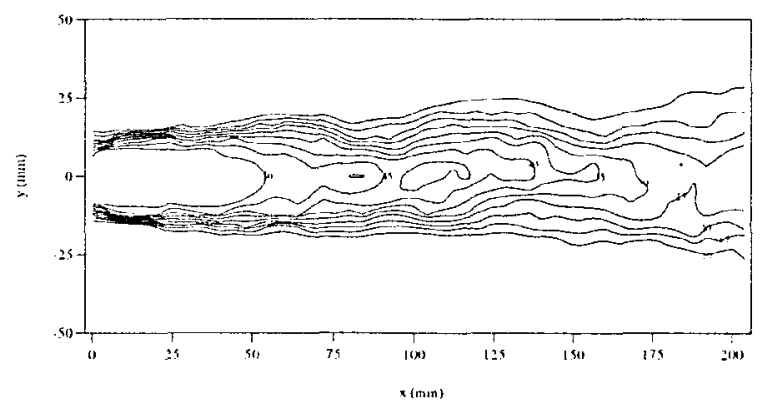

(b)

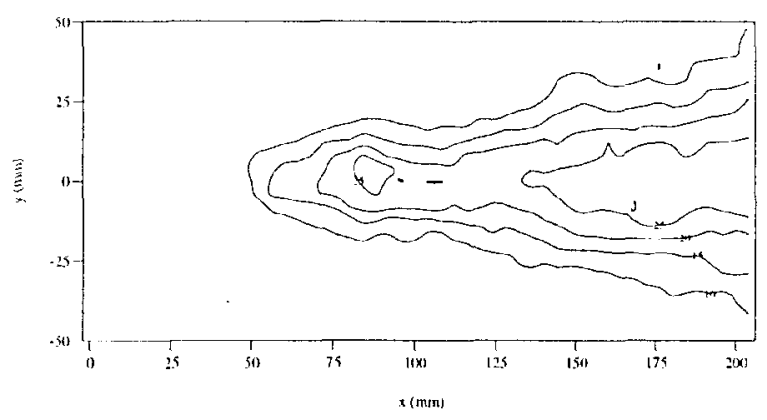

(c)

Figure 12. Streamwise Velocity Component Contours of Averaged PIV Velocity Fields. $U_{c}=50 \mathrm{~cm} / \mathrm{s}, U_{c}=5 \mathrm{~cm} / \mathrm{s}$, $\mathrm{u}_{\mathrm{a}}^{\prime} / \mathrm{U}_{a}=10 \%$. (a) Vertical plane through the jet centerline, (b) horizontal plane through jet centerline, (c) horizontal plane at $z=19 \mathrm{~mm}$. Contour levels are shown in $\mathrm{cm} / \mathrm{s}$. 


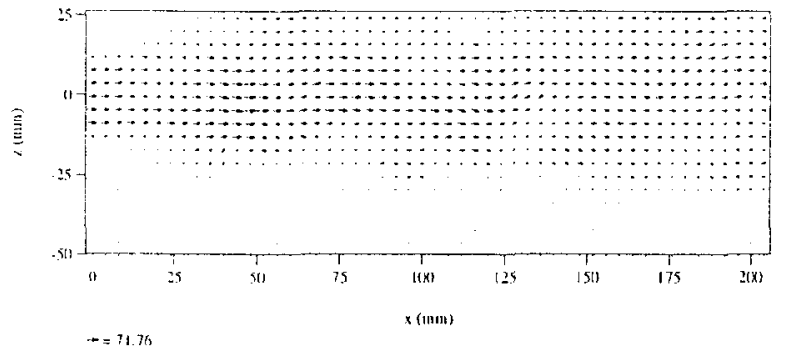

(a)

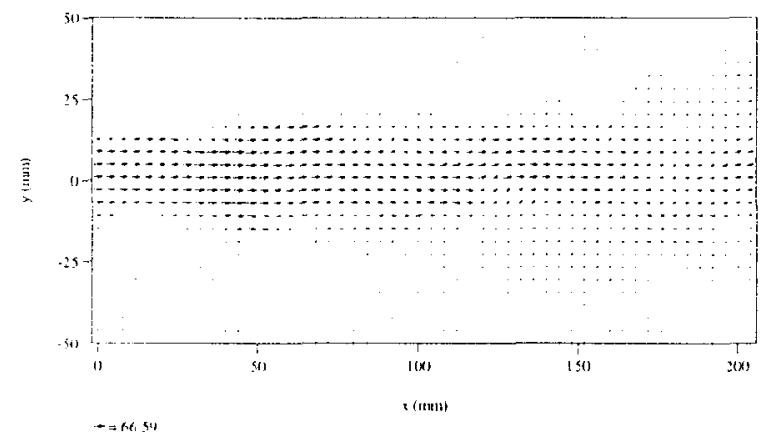

(b)

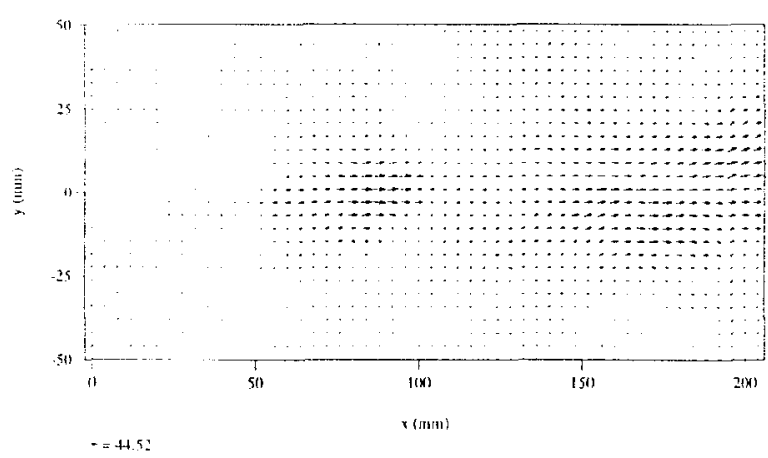

(c)

Figure 13. Vector Plots of Instantaneous Velocity Field in Pulsed Jet, $\Phi=90^{\circ}$. (a) Vertical plane through the jet centerline, (b) horizontal plane through jet centerline, (c) horizontal plane at $z=19 \mathrm{~mm}$. Units of reference vector are $\mathrm{cm} / \mathrm{s}$.

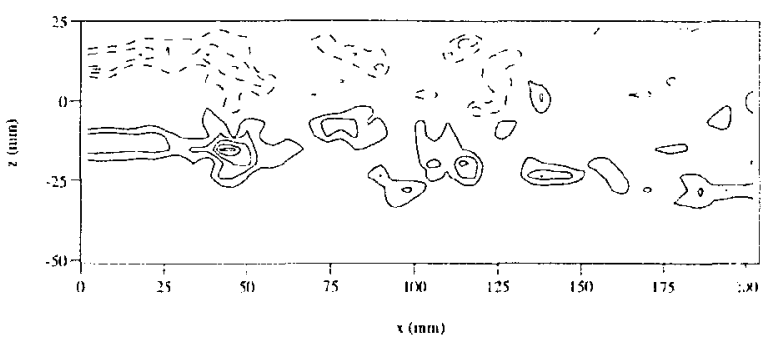

(a)

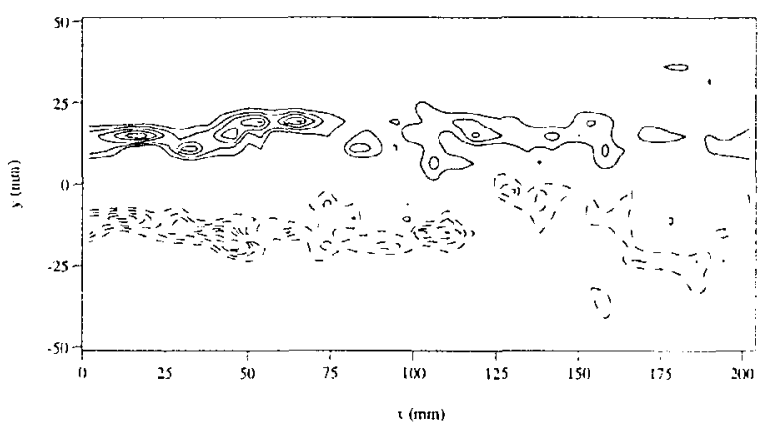

(b)

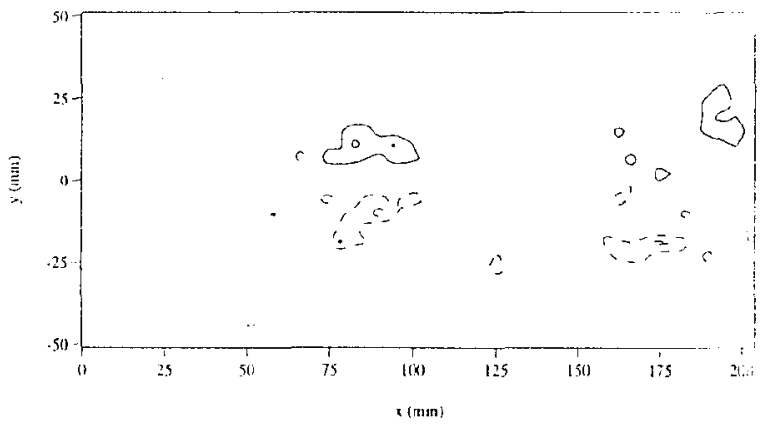

(c)

Figure 14. Contour Plots of Instantaneous Vorticity Field in Pulsed Jet, $\Phi=90^{\circ}$. (a) Vertical plane through the jet centerline, (b) horizontal plane through jet centerline, (c) horizontal plane at $z=19 \mathrm{~mm}$. Contour lines start at $\pm 20 \mathrm{I} / \mathrm{s}$ and are plotted every $20 \mathrm{l} / \mathrm{s}$. Dashed lines represent negalive vorticity. 


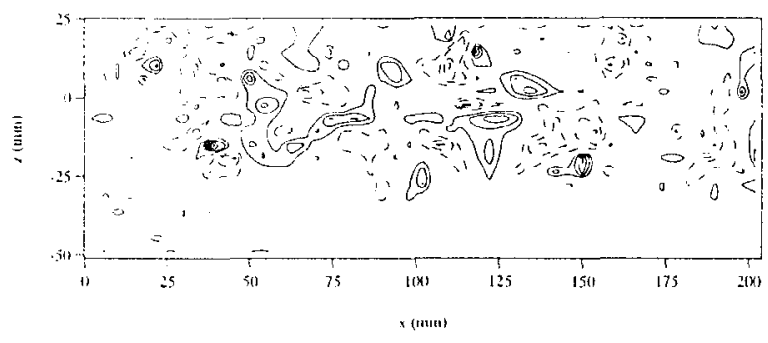

(a)

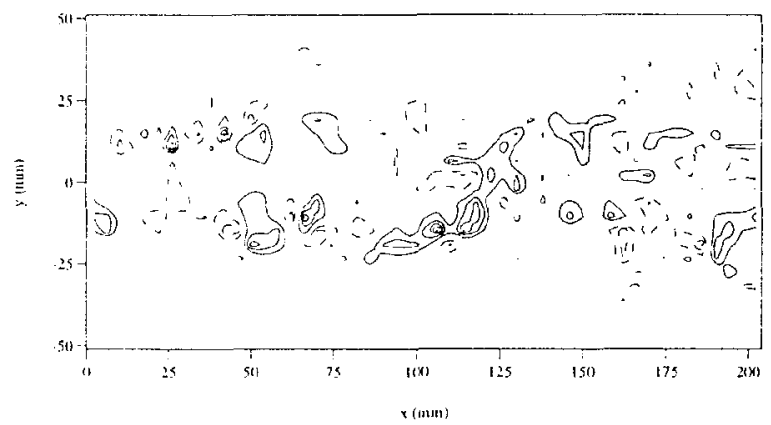

(b)

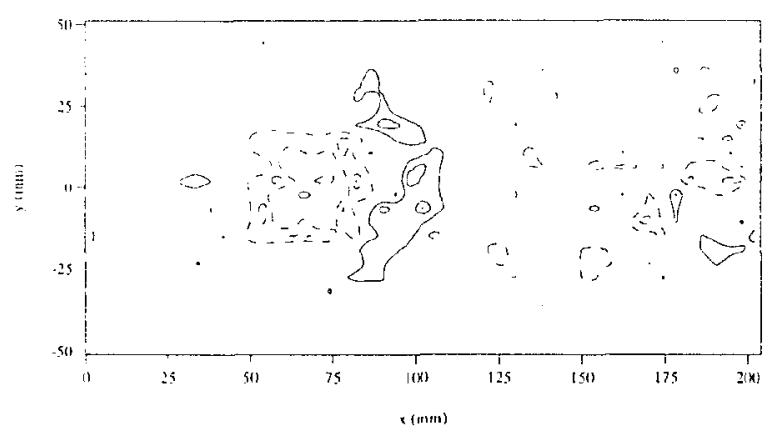

(c)

Figure 15. Contour Plots of Instantaneous Strain Ratc Field in Pulsed Jet. $\Phi=90^{\circ}$. (a) Vertical plane through the jet centerline, (b) horizontal plane through jet centerline. (c) horizontal plane at $z=19 \mathrm{~mm}$. Contour lines start at $\pm 101 / \mathrm{s}$ and are plotted every $10 \mathrm{l} / \mathrm{s}$. Dashed lines represent negative strain rate.

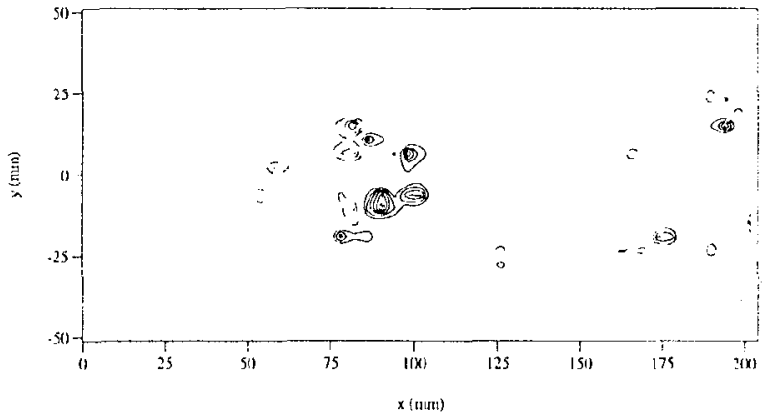

(a)

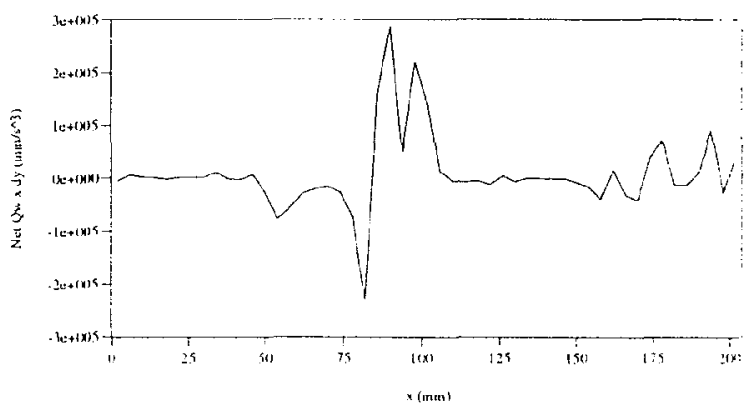

(b)

Figure 16. Enstrophy Production at the Free Surface, $\Phi=90^{\circ}$. (a) Contour plots of enstrophy production $\left(Q_{\omega}\right)$ in a horizontal plane near the free-surface $(6.4 \mathrm{mmi}$ below). Contour lines start at $\pm 5,000 \mathrm{l} / \mathrm{s}^{3}$ and are spaced evisy $5,000 \mathrm{l} / \mathrm{s}^{\prime}$. Solid lines represent enstrophy production, dashed lines reduction. (b) Streamwise variation of total enstrophy production per unit length. Values are obtained by summing across the flow. Note the peaks and valleys corresponding to locations of surface normal vorticity. 
Copyright (c)1997, American Institute of Aeronautics and Astronautics, Inc.

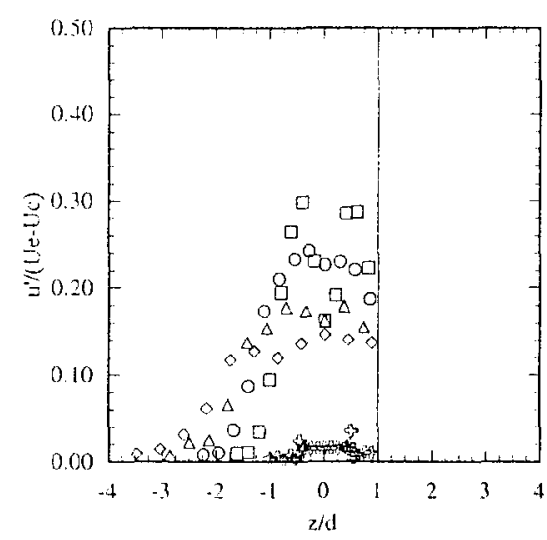

(a)

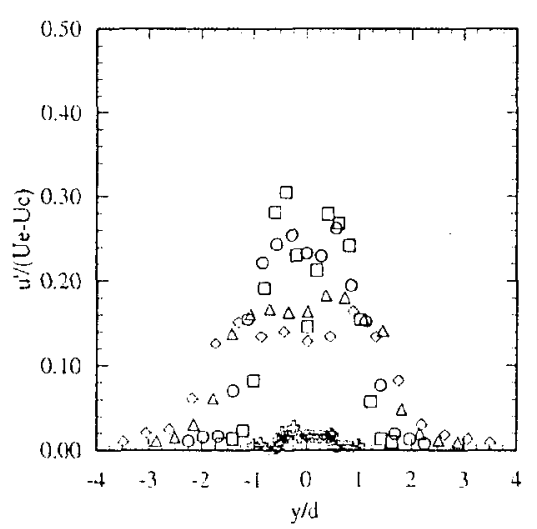

(b)

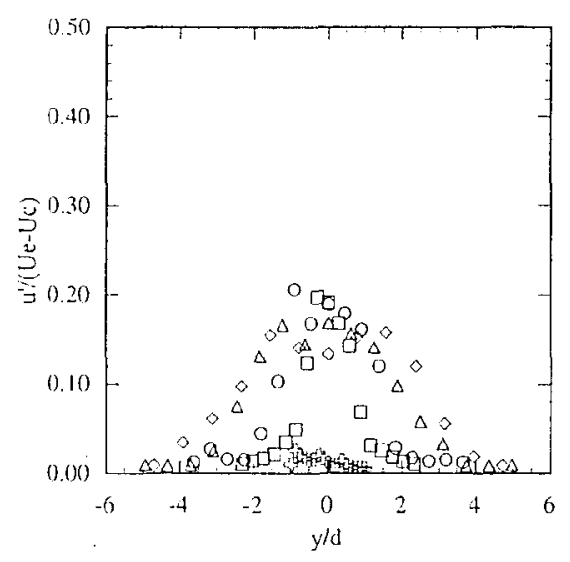

(c)

Figure 17. Hotfilm Fluctuating Velocity Data for Steady Flow. $U_{0}=50 \mathrm{~cm} / \mathrm{s} . U_{s}=5 \mathrm{~cm} / \mathrm{s}$. (a) Vertical profiles through jet centerline, (b) horizontal profiles through jet centerline, (c) horizontal profiles $6.4 \mathrm{~mm}$ below the surface $(z / \mathrm{h}=0.75)$. 色, $x / d=0 ; \square, x / d=4 ; 0, x / d=8 ; \Delta, x / d=12 ; \diamond, x / d=$ 16.

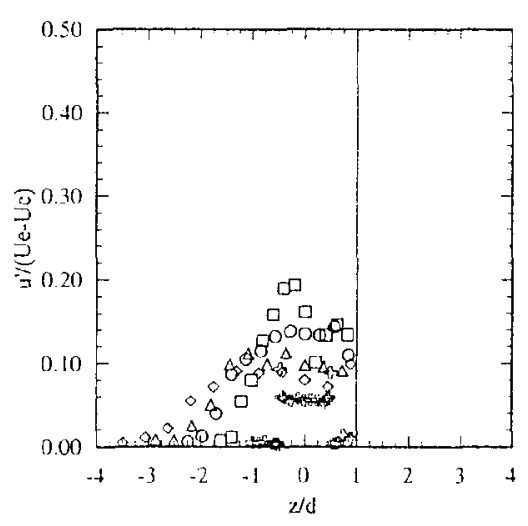

(a)

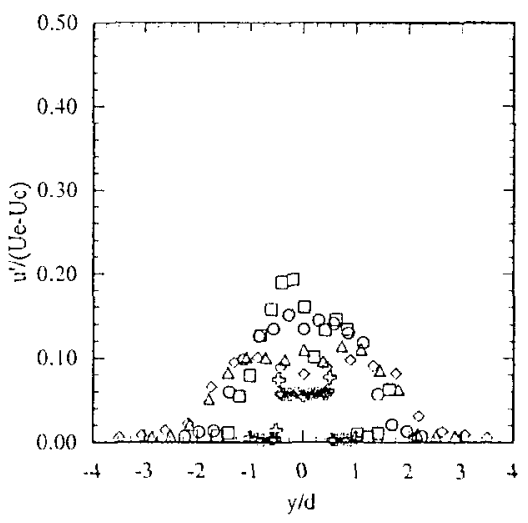

(b)

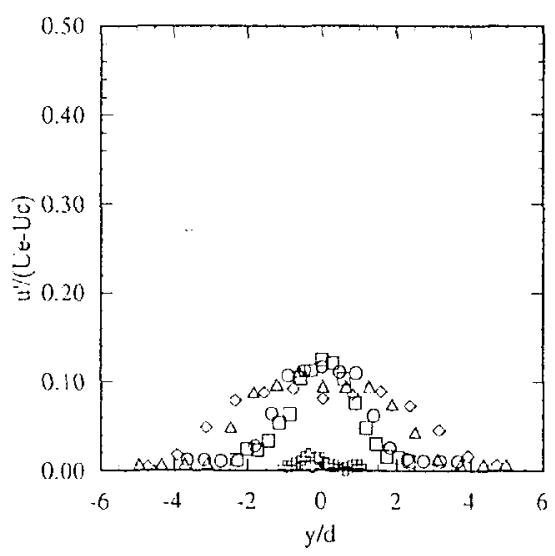

(c)

Figure 18. Hotfilm Fluctuating Velocity Data for Pulsed Flow. $\mathrm{U}_{\mathrm{c}}=50 \mathrm{~cm} / \mathrm{s}, \mathrm{U}_{\mathrm{c}}=5 \mathrm{~cm} / \mathrm{s}, \mathrm{u}_{\mathrm{c}} / \mathrm{U}=10 \%$. (a) Vertical profiles through jet centerlinc, (b) horizontal profiles through jet centerline. (c) horizontal profiles $6.4 \mathrm{~mm}$ below the surface $(z / h=0.75)$. क,$x / d=0 ; \square, x / d=4 ; 0, x / d=3$; $\Delta, x / d=12 ; \diamond, x / d=16$. 